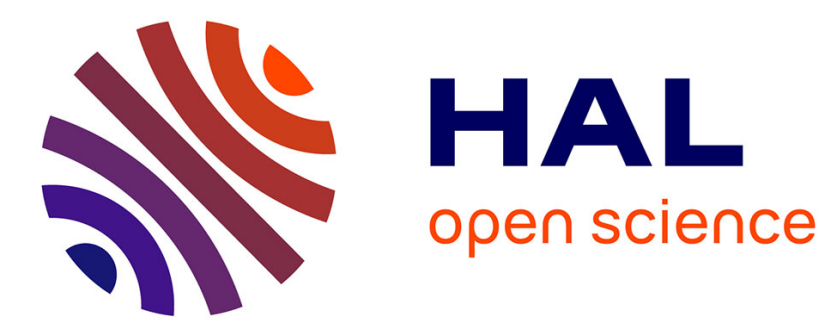

\title{
Reliability assessment of multi-state phased mission system with non-repairable multi-state components
}

\author{
Xiang-Yu Li, Hong-Zhong Huang, Yan-Feng Li, Enrico Zio
}

\section{To cite this version:}

Xiang-Yu Li, Hong-Zhong Huang, Yan-Feng Li, Enrico Zio. Reliability assessment of multi-state phased mission system with non-repairable multi-state components. Applied Mathematical Modelling, 2018, 61, pp.181-199. 10.1016/j.apm.2018.04.008 . hal-01988969

\section{HAL Id: hal-01988969 https://hal.science/hal-01988969}

Submitted on 8 Feb 2019

HAL is a multi-disciplinary open access archive for the deposit and dissemination of scientific research documents, whether they are published or not. The documents may come from teaching and research institutions in France or abroad, or from public or private research centers.
L'archive ouverte pluridisciplinaire HAL, est destinée au dépôt et à la diffusion de documents scientifiques de niveau recherche, publiés ou non, émanant des établissements d'enseignement et de recherche français ou étrangers, des laboratoires publics ou privés. 


\title{
Reliability assessment of multi-state phased mission system with non-repairable multi-state components
}

\author{
Xiang-Yu Li ${ }^{a, b}$, Hong-Zhong Huang ${ }^{\mathrm{a}, *}$, Yan-Feng $\mathrm{Li}^{\mathrm{a}}$, Enrico Zio ${ }^{\mathrm{b}, \mathrm{c}}$ \\ ${ }^{a}$ Center for System Reliability and Safety, School of Mechanical and Electrical Engineering, University of Electronic Science and \\ Technology of China, 611731 Chengdu, China \\ ${ }^{\mathrm{b}}$ Chair System Science and the Energy Challenge, Fondation Électricité de France (EDF), CentraleSupélec, Université Paris Saclay, 91192 \\ Gif-sur-Yvette Cedex, France \\ ${ }^{\mathrm{c}}$ Energy Department, Politecnico di Milano, Milano, Italy
}

\section{A R T I C L E I N F O}

\section{Article history:}

Received 17 December 2017

Revised 12 April 2018

Accepted 18 April 2018

Available online 25 April 2018

\section{Keywords:}

Reliability assessment

Multi-state multivalued decision diagram

(MMDD)

Phased mission system (PMS)

Multi-state system

Semi-Markov process

Monte Carlo simulation

Attitude and Orbit Control System

\begin{abstract}
A B S T R A C T
Phased mission systems (PMSs) like satellites and spacecraft perform their functions over non-overlapping mission periods, called phases. One of the challenges in assessing reliability of PMSs comes from considering the $s$-dependence among phases, and the consideration on the multi-state behavior of components and systems makes the reliability analysis even more difficult. To effectively address this problem, a multi-state multivalued decision diagram algorithm for PMS and a multi-state multi-valued decision diagram model for phased mission system (PMS-MMDD) method is developed for the reliability modelling of non-repairable multi-state components. Based on the Semi-Markov process, a Markov renewal equation-based method is developed to deal with non-exponential multistate components and a numerical method, the trapezoidal integration method, is used to compute the complex integrals in the path probability evaluation. A case study of a multistate attitude and orbit control system in a spacecraft is analyzed to illustrate the proposed PMS-MMDD model and the Markov renewal equation-based evaluation method. The accuracy and computation efficiency of the proposed method are verified by the Monte Carlo simulation method.
\end{abstract}

(c) 2018 Elsevier Inc. All rights reserved.

\section{ACRONYM \\ AOCS Attitude and orbit control system \\ BDD Binary decision diagram \\ CTMC Continuous time Markov chain \\ FT Fault tree \\ MMDD Multi-state multivalued decision diagram \\ PDO Phase dependent operation \\ PMS Phased mission system \\ PMS-BDD Binary decision diagram model for phased mission system \\ PMS-MMDD Multi-state multi-valued decision diagram model for phased mission system \\ SMP Semi-Markov Process}

\footnotetext{
* Corresponding author.

E-mail address: hzhuang@uestc.edu.cn (H.-Z. Huang).
} 


\section{Introduction}

Phased mission systems (PMSs) are typical of the aerospace industry. PMSs perform in multiple, consecutive and nonoverlapping mission durations, known as phases [1]. In the different phases, system is required to accomplish different tasks by different components and system structures in different environments [2]. The degradation process of the systems/components of the PMSs can be described in terms of transitions among multi-states (from perfectly working to totally failure) based on discrete function performance levels [3,4]. Then, the reliability of multi-state phased-mission systems is defined as the probability that the system resides in states above the predefined failure threshold in all phases. Challenges in analyzing the PMS (even binary) are due to two aspects [2]: (1) Dependence among phases: the components failed in one phase will remain in the failure state in the following phases, in non-repairable PMSs. And the component state at beginning of one phase should be equal to its state at the end of last phase; (2) Dynamic behavior among phases: the working components, the system structures and the failure criteria are different due to different mission requirements. In multi-state PMS, the multi-state behaviors renders more complicated the phase dependence and the dynamics leading to the state explosion problem [3].

Most of the works on the PMS reliability focus on binary state PMSs, and their contributions are mainly in two main aspects: (1) improving computational efficiency [5,6]; (2) modelling the PMS with special features, such as external/internal common cause failures [7], imperfect coverage [8] or generalized PMS [9]. The PMS reliability modelling methods employed can be divided into two major categories: simulation approaches [10,11] and analytical approaches [12-16]. Compared to analytical methods, the simulation approaches can deal with more general systems but suffer from high computational costs. The analytical approaches can be further categorized into three types: (1) combinatorial methods, such as the binary decision diagram [13,14] (BDD), the multivalued decision diagram (MDD) [6] and the multi-state multivalued decision diagram (MMDD) [15] methods. These methods can analyze the PMSs very efficiently, especially for large scale systems. However, the combinatorial methods can only handle systems with $s$-independent components. (2) State-space based approaches, such as the continuous time Markov chain (CTMC) [17,18], Semi-Markov Process (SMP) [19] and Petri-nets [20]. State-space based approaches are flexible and powerful in modelling the PMSs with complex dynamic behaviors (e.g., cold standby), such as cold standby, but suffer from the state explosion problem. (3) Modular methods [21], which combined the combinatorial methods with state-space model, merging the advantages of both but limited to some special cases.

In recent years, multi-state systems have been widely studied [22-31] to describe multiple levels of working performance or the degradation process of components and systems. The works on multi-state systems can be categorized by their research objectives: (1) system state probabilities or reliability assessment methods, e.g., by the decision diagram methods [23], the stochastic process methods [24-26] and the universal generation function method [27]; (2) redundancy optimization [28]; (3) parameters estimation [29]; (4) maintenance and warranty research [30], etc. However, all of these works do not consider the multi-phased characteristics of PMSs.

The works on the analysis of multi-state PMS are very few. Recently, the MMDD method has been applied to evaluate the reliability of multi-state PMSs [5]. The MMDD models are generated from the multi-state fault tree model of each phase, then the system MMDD model and disjoint paths for the system are generated by the general MMDD manipulation rules. At last, the CTMC is used to evaluate the probabilities of the disjoint paths from the system MMDD model. In [5], only repairable MS-PMSs are analyzed, but in practical, many systems cannot be repaired, such as satellites or the spacecraft working in the outer space. Part of the paths from the MMDD models generated by the general MMDD manipulation rules may have self-conflict problems in non-repairable systems. For example, one component may be in higher performance states in later phases in the same path because they can be repaired, but these paths are not available in non-repairable PMS. To address this problem, this paper proposes a MMDD algorithm for multi-state PMS and PMS-MMDD model in which the dependency among phases can be fully considered and addressed in the model generation process without additional steps. Furthermore, the more general non-exponential distributions of the state transition times [31,32] are considered, and based on the Semi-Markov Process (SMP) [33,34], a Markov renewal equation-based method is also proposed. A numerical method, the trapezoidal integration method, is used to evaluate the path probabilities.

The paper is organized as follows. Section 2 introduces a simple MS-PMS example and the basic concepts of PMS-BDD method and general MMDD method. In Section 3, phase algebra for the multi-state PMS and the PMS-MMDD model are proposed and described in detail. The MMDD model and disjoint paths generation process are also shown by the PMS example in Section 2. Section 4 proposes the Markov renewal equation-based method for evaluation of path probabilities. In Section 5, a practical example, an attitude and orbit control system (AOCS) of a spacecraft is used to illustrate the proposed PMSMMDD method. Then, the proposed method is compared to the existing path evaluation method. Moreover, the proposed method is compared to the Monte Carlo simulation method under different number of simulation realizations to show its accuracy and computation efficiency. Section 6 gives the conclusions and future works.

\section{MMDD method for non-repairable PMSs}

\subsection{Description of the example PMS}

A simple phased-mission system consisting of three components and three phases is used to show the PMS-MMDD method proposed in this paper. The system consists of three components A, B and C and the state transition graphs for the 
(1) For component $A$

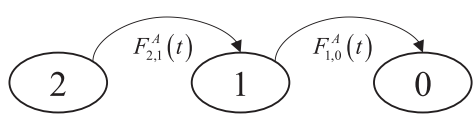

(2) For component $B$

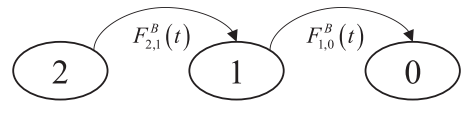

(3) For component $C$

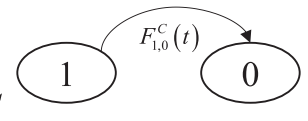

Fig. 1. The state transition graphs for components in the example system.

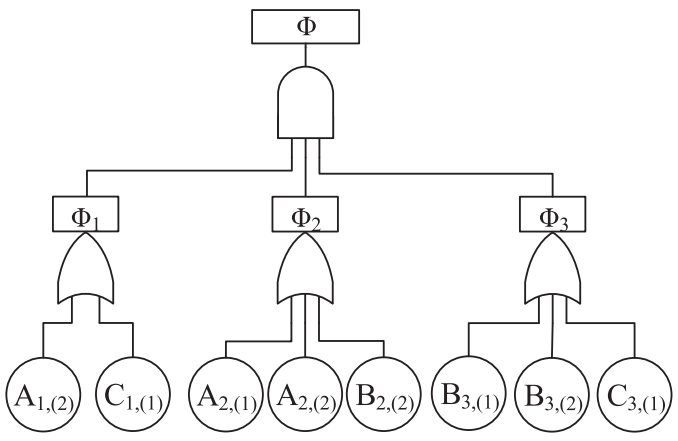

Fig. 2. The multi-state FT model for the example system.

three components are shown in Fig. 1. $F_{i, j}(t)$ represents the cumulative distribution function of the state transition time from state $i$ to state $j$ at time $t$.

The example PMS consists of three consecutive missions: $\tau_{1}, \tau_{2}$ and $\tau_{3}$. Mission $\tau_{1}$ needs component $A$ in state 2 or component $C$ in state 1 ; Mission $\tau_{2}$ needs component $A$ above state 1 or component $B$ in state 2; Mission $\tau_{3}$ needs component $B$ above state 1 or component $C$ in state 1 . The entire mission is successful if all the three consecutive missions are completed successfully. The system structure function for this example PMS is described as,

$$
\Phi=\Phi_{1} \cdot \Phi_{2} \cdot \Phi_{3}=\left(A_{1,(2)}+C_{1,(1)}\right)\left(A_{2,(1)}+A_{2,(2)}+B_{2,(2)}\right)\left(B_{3,(1)}+B_{3,(2)}+C_{3,(1)}\right)
$$

In Eq. (1), each element represents that one component in one specific phase and state, for example, $A_{1,(2)}$ represents that component $A$ is in state 2 in phase 1 . According to Eq. (1), the system multi-state fault tree (FT) model is shown in Fig. 2.

\subsection{Basic concepts of $B D D$ and $M M D D$}

In this section, the basics of the BDD model and its extended MMDD model, as well as the PMS-BDD model for the binary non-repairable PMS, are introduced in details.

\subsubsection{BDD model and PMS-BDD method}

A BDD model is a directed acyclic graph based on the Shannon decomposition. Let $f$ be a Boolean expression for a binary component $\mathrm{A}$, the Boolean function can be expressed as: $f=A \cdot f_{A=1}+\bar{A} \cdot f_{A}=0$. The if-then-else (ite) format is used to express the decomposition and the ite format of component $\mathrm{A}$ is: $f=i t e\left(A, f_{1}, f_{0}\right)$. The entire BDD model is rooted with two sink nodes, 0 and 1, representing the system's failure and success, respectively. Each non-sink node is also labeled with two edges called the 0-edge and 1-edge to represent the Boolean expressions of the component's failure and success, respectively.

The BDD model is generated recursively by the BDD manipulation with all the components' Boolean expressions,

$$
\begin{aligned}
& g \triangleleft h=\text { ite }\left(x, G_{1}, G_{2}\right) \text { ite }\left(y, H_{1}, H_{2}\right)
\end{aligned}
$$

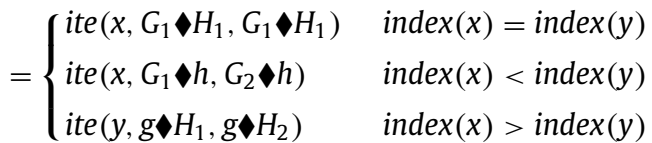

$g$ and $h$ are Boolean expressions for components $x$ and $y$, respectively. represents the logic expressions (OR, AND) and the index denotes the predefined order of the variables [8]. 
Table I

Phase algebra for the BDD model $(1 \leq i<j<n)$.

\begin{tabular}{ll}
\hline$A_{i,(1)} \bullet A_{j,(1)} \rightarrow A_{j,(1)}$ & $A_{i,(1)}+A_{j,(1)} \rightarrow A_{i,(1)}$ \\
$\bar{A}_{i,(1)} \bullet \bar{A}_{j,(1)} \rightarrow \bar{A}_{i,(1)}$ & $\bar{A}_{i,(1)}+\bar{A}_{j,(1)} \rightarrow \bar{A}_{j,(1)}$ \\
$\bar{A}_{i,(1)} \bullet A_{j,(1)} \rightarrow 0$ & $\bar{A}_{i,(1)}+A_{j,(1)} \rightarrow 1$ \\
\hline
\end{tabular}

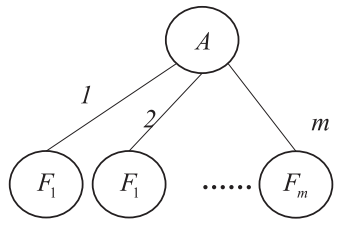

(a) General MMDD

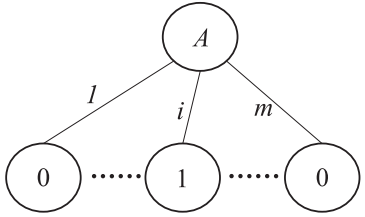

(b) basic MMDD event

Fig. 3. MMDD models.

The PMS-BDD method is proposed in [8] to deal with the dependence among phases in the PMS with the BDD model. Assume that component $A$ is a binary components working in $n$ phases. In the non-repairable binary PMS, according to the relationship of the same component in different phases, phase algebra for binary PMS is proposed in [8], as shown in Table I.

In Table I, component $A$ only has two states: $A_{i,(1)}=1$ means component $A$ works normal in phase $i$ and $\bar{A}_{i,(1)}=1$ means that component $\mathrm{A}$ is in failure state in phase $i$. In The physical meaning of the elements in the left column are: (1) $A_{i,(1)} \bullet$ $A_{j,(1)} \rightarrow A_{j,(1)}$, Component $A$ is working in phase $i$ and phase $j$ implies component A works in phase $j .(2) \bar{A}_{i,(1)} \cdot \bar{A}_{j,(1)} \rightarrow \bar{A}_{i,(1)}$, component $\mathrm{A}$ is failed in phase $i$ and phase $j$ implies component $\mathrm{A}$ is failed in phase $i$. (3) $\bar{A}_{i,(1)} \cdot A_{j,(1)} \rightarrow 0$, component $A$ is failed in phase $i$ and works in phase $j$ is not possible.

With this phase algebra and the backward phase dependent operation (PDO), two Boolean expressions $E_{i}=$ ite $\left(\bar{A}_{i,(1)}, G_{1}, G_{2}\right)$ and $E_{j}=$ ite $\left(\bar{A}_{j,(1)}, H_{1}, H_{2}\right)$, representing the behaviors of component $A$ in phase $i$ and phase $j$ respectively, the BDD manipulation for the non-repairable PMS can be changed as,

$$
\text { ite }\left(\bar{A}_{i,(1)}, G_{1}, G_{2}\right) \triangleleft i t e\left(\bar{A}_{j,(1)}, H_{1}, H_{2}\right)=\operatorname{ite}\left(\bar{A}_{j,(1)}, E_{i} \diamond H_{1}, G_{2} \triangleleft H_{2}\right)
$$

With the backward PDO and the PMS-BDD method, the cancellation of the common components can be automatically done in the model generation process, which can make the system BDD model smaller. More information and details about the BDD model and PMS-BDD method can be found in [15].

\subsubsection{MMDD model}

MMDD is a directed acyclic graph extended from BDD and based on Shannon's decomposition for the symbolic representation and manipulation of multi-valued logic functions [1]. Like the ite format in the BDD operation, the case format of $\operatorname{logic}$ expression $F$ for a multi-state component $A$ with $m$ states is defined as [15],

$$
\begin{aligned}
F & =A_{1} \cdot F_{x_{A}=1}+A_{2} \cdot F_{x_{A}}=2+\cdots+A_{m} \cdot F_{x_{A}}=m \\
& =\operatorname{case}\left(A, F_{x_{A}}=1, F_{x_{A}}=2, \ldots, F_{x_{A}}=m\right. \\
& =\operatorname{case}\left(A, F_{1}, F_{2}, \ldots, F_{m}\right)
\end{aligned}
$$

The MMDD format of Eq. (4) and the basic event $A_{i}$, which means the component is in state $i(1 \leq i \leq m)$, are respectively shown in Fig. 3(a) and (b).

With the basic MMDD event for each state of each component, the system MMDD model can be generated by the MMDD manipulation rules, which is also extended from the BDD manipulation rules, shown as [15],

$$
\begin{aligned}
& g \checkmark h=\operatorname{case}\left(x, G_{1}, \ldots G_{m}\right) \operatorname{casey}\left(y, H_{1}, \ldots H_{m}\right) \\
& = \begin{cases}\operatorname{case}\left(x, G_{1} H_{1}, \ldots, G_{m} H_{m}\right) & \text { index }(x)=i n d e x(y) \\
\operatorname{case}\left(x, G_{1}\right. & \text { index }(x)<i n d \operatorname{lin}(y) \\
\operatorname{case}\left(y, g \bullet H_{1}, \ldots, g H_{m}\right) & \text { index }(x)>\operatorname{index}(y)\end{cases}
\end{aligned}
$$

where $g$ and $h$ are Boolean expressions with multi-valued inputs and represents the logic operations (OR, AND). The index means the predefined order of the variables.

In the system MMDD model, there are two sink nodes ' 1 ' and ' 0 ', representing the component being/not being in one specific state. Each path from the root node to the sink node ' 1 ' represents the combinations of components' states that the system is in this specific state. 
Table II

The phase algebra for the multi-state PMS $(1 \leq i<j \leq N)$.

\begin{tabular}{ll}
\hline$A_{i,(m)} \bullet A_{j,(m)} \rightarrow A_{j,(m)}$ & $A_{i,(m)}+A_{j,(m)} \rightarrow A_{i,(m)}$ \\
$A_{i,(1)} \bullet A_{j,(1)} \rightarrow A_{i,(1)}$ & $A_{i,(1)}+A_{j,(1)} \rightarrow A_{j,(1)}\left(1 \leq S_{j}<S_{i} \leq m\right)$ \\
$A_{i,\left(S_{i}\right)} \bullet A_{j,\left(S_{j}\right)} \rightarrow 0\left(1 \leq S_{i}<S_{j} \leq m\right)$ & $A_{i,\left(S_{i}\right)}+A_{j,\left(S_{j}\right)} \rightarrow 1 \quad\left(1 \leq{ }^{2}\right.$ \\
\hline
\end{tabular}

\section{MMDD method for non-repairable PMS}

\subsection{The phase algebra for the MMDD model}

In previous research on the multi-state PMS, only repairable components have been considered and phase dependence among components amounts to ensuring that states at the end of the former phase remains the same as the beginning of the successive phase. However, in non-repairable multi-state PMS, the dependency among phases is more complicated. For example, the components' state cannot be better in the latter phases than in the former phases. A phase algebra for PMS and PMS-MMDD method are proposed to address this problem in this section.

\subsubsection{The phase algebra for PMS}

The phase algebra for the BDD method is extended to the MMDD model and the phase algebra for the multi-state PMS is developed. Assume that component $A$ is a component with $m$ states and works in $N$ phases ( $m$ is the best state and 1 is the worst state). Then, the phase algebra for the multi-state PMS is proposed in Table II. Based on the rules of phase algebra for binary components shown in Table I and the general MMDD model, the rules of phase algebra for the non-repairable multi-state components are developed and shown in Table II.

$A_{i,(m)}=1$ means component $A$ is in state $m$ and $A_{i,(m)}=0$ means component $A$ is not in state $m ; S_{i}$ and $S_{j}$ in the last line represent the states of component $A$ in phase $i$ and phase $j$, respectively.

The physical meaning of the phase algebra for the multi-state PMS in Table II are:

(1) $A_{i,(m)} \bullet A_{j,(m)} \rightarrow A_{j,(m)}$, component $A$ is in the perfect state $m$ both in phase $i$ and phase $j$ implies component $A$ is in state $m$ in phase $j$.

(2) $A_{i,(1)} \bullet A_{j,(1)} \rightarrow A_{i,(1)}$, component $A$ is in worst state 1 both in phase $i$ and phase $j$ implies component $A$ is in worst state 1 in phase $i$.

(3) $A_{i,\left(S_{i}\right)} \bullet A_{j,\left(S_{j}\right)} \rightarrow 0\left(1 \leq S_{i}<S_{j} \leq m\right)$, component $A$ is in state $S_{i}$ in phase $i$ and is in state $S_{j}$ in phase $j$ simultaneously is not exist.

(4) $A_{i,(m)}+A_{j,(m)} \rightarrow A_{i,(m)}$, component $A$ is in the perfect state $m$ in phase $i$ or phase $j$ implies component $A$ is in state $m$ in phase $i$.

(5) $A_{i,(1)}+A_{j,(1)} \rightarrow A_{j,(1)}$, component $A$ is in state 1 in phase $i$ or phase $j$ implies component $A$ is in state 1 in phase $j$.

(6) $A_{i,\left(S_{i}\right)}+A_{j,\left(S_{j}\right)} \rightarrow 1\left(1 \leq S_{j}<S_{i} \leq m\right)$, has no physical meaning if the components are not repairable.

\subsubsection{MMDD operation for phase algebra}

With the phase algebra for multi-state PMS, the general MMDD manipulation rules [5] of the same component in different phases can be changed into MMDD manipulation rules for multi-state PMS. To apply the special logic relations shown in Table II in the MMDD model generation, a special MMDD operation, PDO, is derived for the relations. According to [8], there are two kinds of PDO: (1) forward PDO, the order of the variables are the same as the phase order; (2) backward PDO, the order of the variables is reverse of the phase order.

Assume that component $A$ with $m$ states works both in phase $i$ and phase $j(i<j)$, and the case format of component $A$ in phase $i$ and phase $j, E_{i}$ and $E_{j}$, can be written as,

$$
\begin{aligned}
& E_{i}=\operatorname{case}\left(A_{i},\left(E_{i}\right)_{A_{i,(m)}}, \ldots,\left(E_{i}\right)_{A_{i,(1)}}\right)=\operatorname{case}\left(A_{i}, G_{m}, \ldots, G_{1}\right) \\
& E_{j}=\operatorname{case}\left(A_{j},\left(E_{j}\right)_{A_{j,(m)}}, \ldots,\left(E_{j}\right)_{A_{j,(1)}}\right)=\operatorname{case}\left(A_{j}, H_{m}, \ldots, H_{1}\right)
\end{aligned}
$$

where $G_{m}, H_{m}=1$ means component $A$ is in state $m$ in phase $i$ and phase $j . G_{m}, H_{m}=0$ means component $A$ is not in state $m$ in phase $i$ and phase $j$.

Integrating the phased algebra for MMDD and the general MMDD manipulation rule, the MMDD manipulation rule for PMS can be derived, shown as,

(1) for the forward PDO $\left(\operatorname{index}\left(A_{i}<A_{j}\right)(i<j)\right)$, the proposed MMDD manipulation rule for the multi-state PMS is,

$$
\begin{aligned}
& \operatorname{case}\left(A_{i}, G_{m}, \ldots \ldots, G_{1}\right) \backslash \operatorname{case}\left(A_{j}, H_{m}, \ldots \ldots, H_{1}\right) \\
& \quad=\operatorname{case}\left(A_{i}, G_{m} \diamond E_{j, 1}, G_{m-1} \diamond E_{j, 2}, \ldots \ldots, G_{2} \triangleright E_{j, m-1}, G_{1} \diamond H_{1},\right)
\end{aligned}
$$

where the $E_{j, n}=\operatorname{case}(A_{j}, \underbrace{0, \ldots, 0}_{n-1}, H_{m-n+1}, \ldots, H_{2}, H_{1}), \quad 1 \leq n \leq m$. 


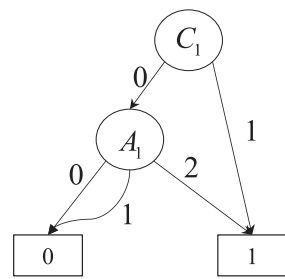

(Phase 1)

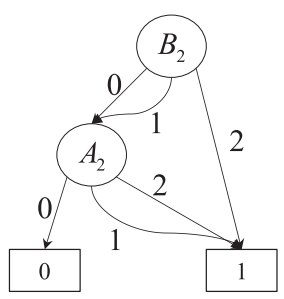

(Phase 2)

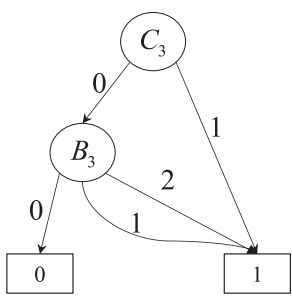

(Phase 3)

Fig. 4. The MMDD model for each phase.

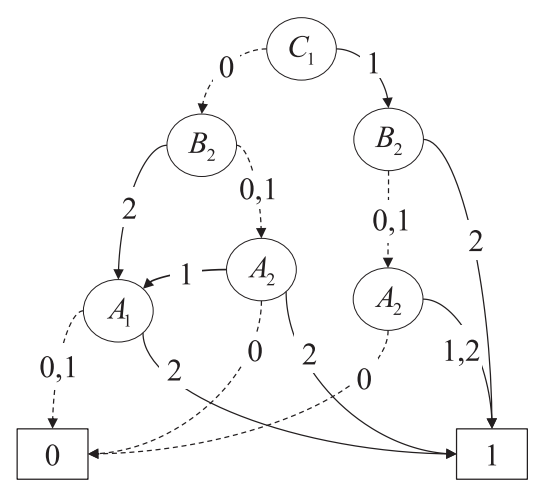

Fig. 5. The grouped MMDD model for the first two phases of the example PMS.

(2) for the backward PDO $\left(\operatorname{index}\left(A_{i}>A_{j}\right)(i<j)\right)$, the proposed MMDD manipulation rule for the multi-state PMS is,

$$
\begin{aligned}
& \operatorname{case}\left(A_{i}, G_{m}, \ldots, G_{1}\right) \backslash \operatorname{case}\left(A_{j}, H_{m}, \ldots, H_{1}\right) \\
& =\operatorname{case}\left(A_{j}, G_{m} \diamond H_{m}, E_{i, 2} \triangleleft H_{m-1}, \ldots, E_{i, m} \diamond H_{1}\right)
\end{aligned}
$$

where the $E_{i, n}=\operatorname{case}(A_{i}, G_{m}, G_{m-1}, \ldots, G_{m-n+1}, \underbrace{0, \ldots, 0}_{m-n}), 1 \leq n \leq m$.

The proofs of Eqs. (8) and (9) are given in Appendix.

By the MMDD manipulation rule for the multi-state PMS, the self-conflict combination of component states in different phases can be automatically cancelled in the system MMDD model generation process without additional operation.

\subsection{MMDD model generation procedure of the Multi-state PMS}

Using the example of multi-state PMS shown in Fig. 2, the PMS-MMDD method is demonstrated to show the model generation procedure and it can be divided into several steps,

Step 1: each bottom element in the multi-state FT model is represented by a case function. For example, the elements in phase $1, A_{1,(2)}$ and $C_{1,(1)}$ can be represented by $A_{1,(2)}=\operatorname{case}\left(A_{1}, 1,0,0\right)$ and $C_{1,(1)}=\operatorname{case}\left(C_{1}, 1,0\right)$, respectively.

Step 2: according to the MMDD manipulation rules shown in Eq. (5), the variables for the same components within the same phase can be grouped and the MMDD model for each phase can be constructed by manipulating the case function of the working components by the ordinary MMDD manipulation rules. The MMDD models for the three phases of the example PMS are shown in Fig. 4.

Step 3: integrate the MMDD models for all phases by the proposed PMS-MMDD model. In general, the size of the system model is smaller by the backward PDO. By using the backward PDO, the variables ordering $C_{3}<C_{1}<B_{3}<B_{2}<A_{2}$ $<A_{1}$; the system MMDD model for the first two phases and all three phases are shown in Figs. 5 and 6, respectively.

In Figs. 5 and 6, the solid lines represent the grouped working states and the dashed lines represent the grouped the failure states. In this MMDD model, each path from the root node $C_{3}$ to the sink node ' 1 ' represents the system working normal in all three phases. 


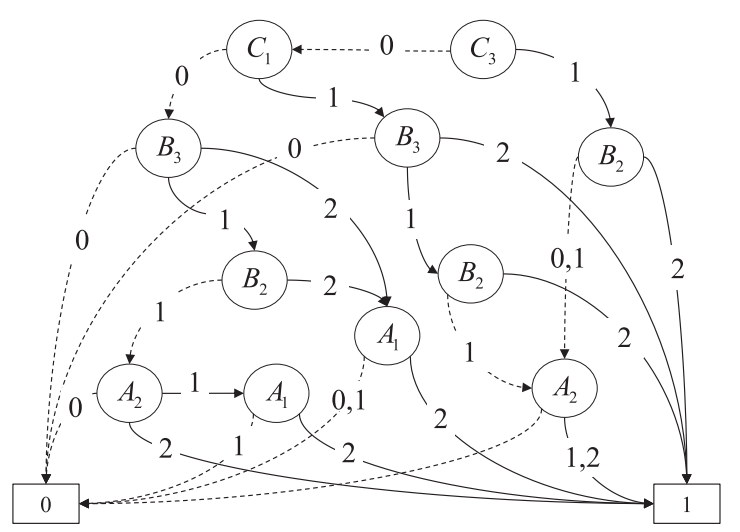

Fig. 6. The grouped MMDD model for all three phases of the example PMS.

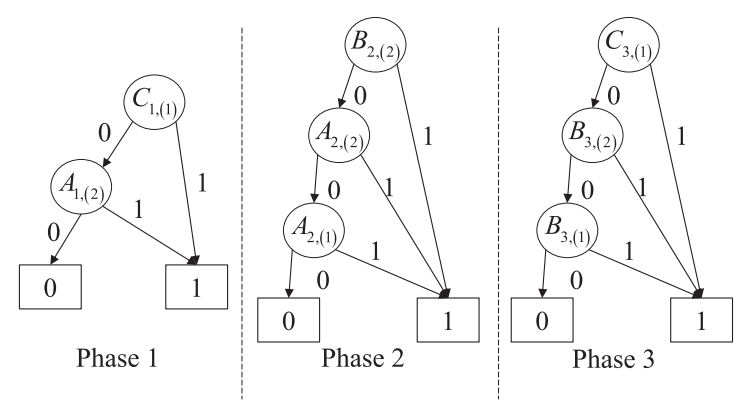

Fig. 7. The BDD model for all three phases of the example PMS.

According to Fig. 6, there are totally 11 paths that the example system can work normally in all 3 phases, shown as,

$$
\begin{aligned}
& \eta_{1}=C_{3,(1)} B_{2,(2)}, \quad \eta_{2}=C_{3,(1)} B_{2,(0)} A_{2,(1)}, \quad \eta_{2}=C_{3,(1)} B_{2,(0)} A_{2,(2)}, \\
& \eta_{3}=C_{3,(1)} B_{2,(1)} A_{2,(1)}, \quad \eta_{4}=C_{3,(1)} B_{2,(1)} A_{2,(2)}, \quad \eta_{5}=C_{3,(0)} C_{1,(1)} B_{3,(2)}, \\
& \eta_{6}=C_{3,(0)} C_{1,(1)} B_{3,(1)} B_{2,(2)}, \quad \eta_{7}=C_{3,(0)} C_{1,(1)} B_{3,(1)} B_{2,(1)} A_{2,(1,2)} \\
& \eta_{8}=C_{3,(0)} C_{1,(0)} B_{3,(2)} A_{1,(2)}, \quad \eta_{9}=C_{3,(0)} C_{1,(0)} B_{3,(1)} B_{2,(2)} A_{1,(2)} \\
& \eta_{10}=C_{3,(0)} C_{1,(0)} B_{3,(1)} B_{2,(1)} A_{2,(2)}, \quad \eta_{11}=C_{3,(0)} C_{1,(0)} B_{3,(1)} B_{2,(1)} A_{2,(1)} A_{1,(2)}
\end{aligned}
$$

where $C_{i,\left(S_{j}\right)}$ means component $C$ stays in states $S_{j}$ in phase $i$.

\subsection{Comparison to existing methods}

In this section, the proposed PMS-MMDD model is compared to the existing PMS-BDD method [14] and the general MMDD method [5], which have been used in modelling multi-state systems.

\subsubsection{Comparison to PMS-BDD model}

The PMS-BDD model is proposed in [8] and widely used in the modelling of binary-state PMS, and also in the modelling of multi-state systems [15]. The model construction procedure of the multi-state PMS by the PMS-BDD model can be divided into various steps as follows,

Step 1. Build the multi-state fault tree model for each phase, and transform the fault tree model into BDD model for the three phases, shown as Fig. 7.

In Fig. 7, each node represents that one component in one specific phase and state. For example, $B_{3,(2)}$ means state 2 of component B in phase 3.

Step 2. Integrate the BDD models for all phases in Fig. 7 into the system BDD models according to the PMS-BDD method. As mentioned above, the backward PDO can make the system BDD model smaller, so the variables ordering is: $C_{3,(1)}<C_{1,(1)}$ $<B_{3,(2)}<B_{3,(1)}<B_{2,(2)}<A_{2,(2)}<A_{2,(1)}<A_{1,(2)}$. Then, the system BDD model is generated by the PMS-BDD model, as shown in Fig. 8.

In Fig. 8, the line ' 1 ' of each node (solid line) indicates that the component is in the state and phase and line ' 0 ' (dashed line) indicates that the component is not in the state of this phase. Each path from the root node $C_{3,1}$ to the sink node ' 1 ' represents the fact that the system works normally in all three phases. 


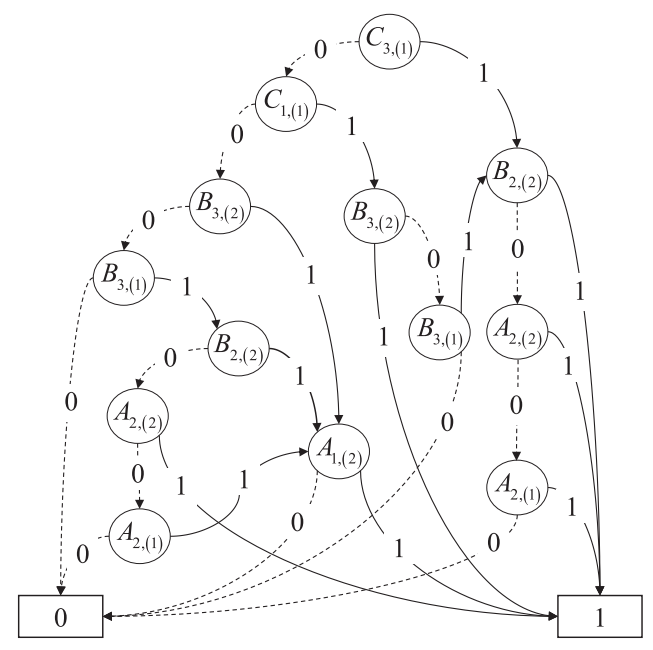

Fig. 8. The system BDD model for the example PMS.

According to Fig. 8, there are totally 11 paths for the system working normally in all 3 phases,

$$
\begin{aligned}
& \eta_{1}^{\prime}=C_{3,(1)} B_{2,(2)}, \quad \eta_{2}^{\prime}=C_{3,(1)} \bar{B}_{2,(2)} A_{2,(2)}, \quad \eta_{3}^{\prime}=C_{3,(1)} \bar{B}_{2,(2)} \bar{A}_{2,(2)} A_{2,(1)} \\
& \eta^{\prime}{ }_{4}=\bar{C}_{3,(1)} C_{1,(1)} B_{3,(2)}, \quad \eta^{\prime}{ }_{5}=\bar{C}_{3,(1)} C_{1,(1)} \bar{B}_{3,(2)} B_{3,(1)} B_{2,(2)} \\
& \eta^{\prime}{ }_{6}=\bar{C}_{3,(1)} C_{1,(1)} \bar{B}_{3,(2)} B_{3,(1)} \bar{B}_{2,(2)} A_{2,(2)}, \quad \eta^{\prime}=\bar{C}_{3,(1)} C_{1,(1)} \bar{B}_{3,(2)} B_{3,(1)} \bar{B}_{2,(2)} \bar{A}_{2,(2)} A_{2,(1)} \\
& \eta^{\prime}{ }_{8}=\bar{C}_{3,(1)} \bar{C}_{1,(1)} B_{3,(2)} A_{1,(2)}, \quad \eta_{9}^{\prime}=\bar{C}_{3,(1)} \bar{C}_{1,(1)} \bar{B}_{3,(2)} B_{3,(1)} B_{2,(2)} A_{1,(2)} \\
& \eta^{\prime}{ }_{10}=\bar{C}_{3,(1)} \bar{C}_{1,(1)} \bar{B}_{3,(2)} B_{3,(1)} \bar{B}_{2,(2)} A_{2,(2)}, \quad \eta^{\prime}{ }_{11}=\bar{C}_{3,(1)} \bar{C}_{1,(1)} \bar{B}_{3,(2)} B_{3,(1)} \bar{B}_{2,(2)} \bar{A}_{2,(2)} A_{2,(1)} A_{1,(2)}
\end{aligned}
$$

In Eq. (11), $\bar{B}_{2,(2)}$ means component $B$ is not at state 2 in phase 2 , and $\bar{B}_{2,(2)}$ is equal to $B_{2,(1,0)}$ in Eq. (10). After analyzing, we can find that the paths in Eq. (11) can correspond to the paths in Eq. (10).

In the BDD model, each node can only describe that one component in one specific state or not, so more nodes are necessary to represent the multi-state component in one specific state. On the contrary, by the proposed PMS-MMDD model, the system model can be generated by less nodes, the system model is simpler and the system disjoint paths can be generated in simpler way.

\subsubsection{Comparison to general MMDD model}

The general MMDD model is used to model the multi-state PMS in [5], in which all the multi-state components are repairable. This method do not consider the second dependency among the phases in the model construction process, and an additional steps is necessary after generating the paths if the components are non-repairable. The model construction procedure of the non-repairable multi-state PMS by the general MMDD model can be divided into several steps, as follows,

Step 1 and step 2 are the same as the first two steps of the PMS-MMDD model construction process in Section 3.2.

Step 3. Using the same variables ordering as Section 3.2, $C_{3}<C_{1}<B_{3}<B_{2}<A_{2}<A_{1}$, integrate the MMDD mode for each phase into the system MMDD model by the general MMDD manipulation rules given in Eq. (5). Then, the system MMDD is shown in Fig. 9.

From Fig. 9, we can get the 25 disjoint paths from the root node ' $C_{3}$ ' to the sink node ' 1 ' in the MMDD model,

$$
\begin{array}{ll}
\eta^{\prime \prime}{ }_{1}=C_{3,(1)} C_{1,(1)} B_{2,(2)}, & \eta^{\prime \prime}{ }_{2}=C_{3,(1)} C_{1,(1)} B_{2,(0)} A_{2,(1)}, \\
\eta^{\prime \prime}{ }_{3}=C_{3,(1)} C_{1,(1)} B_{2,(0)} A_{2,(2)}, & \eta^{\prime \prime}{ }_{4}=C_{3,(1)} C_{1,(1)} B_{2,(1)} A_{2,(1)}, \\
\eta^{\prime \prime}{ }_{5}=C_{3,(1)} C_{1,(1)} B_{2,(1)} A_{2,(2)}, & \eta^{\prime \prime}{ }_{6}=C_{3,(0)} C_{1,(1)} B_{3,(1)} B_{2,2} \\
\eta^{\prime \prime}{ }_{7}=C_{3,(0)} C_{1,(1)} B_{3,(2)} B_{2,(2)}, & \eta^{\prime \prime}{ }_{3}=C_{3,(0)} C_{1,(1)} B_{3,(1)} B_{2,(0)} A_{2,(1)}, \\
\eta^{\prime \prime}{ }_{9}=C_{3,(0)} C_{1,(1)} B_{3,(1)} B_{2,(0)} A_{2,(2)}, & \eta^{\prime \prime}{ }_{10}=C_{3,(0)} C_{1,(1)} B_{3,(1)} B_{2,(1)} A_{2,(1)}, \\
\eta^{\prime \prime}{ }_{11}=C_{3,(0)} C_{1,(1)} B_{3,(1)} B_{2,(1)} A_{2,(2)}, & \eta^{\prime \prime}{ }_{12}=C_{3,(0)} C_{1,(1)} B_{3,(2)} B_{2,(0)} A_{2,(1)}, \\
\eta^{\prime \prime}{ }_{13}=C_{3,(0)} C_{1,(1)} B_{3,(2)} B_{2,(0)} A_{2,(2)}, & \eta^{\prime \prime}{ }_{14}=C_{3,(0)} C_{1,(1)} B_{3,(2)} B_{2,(1)} A_{2,(1)}, \\
\eta^{\prime \prime}{ }_{15}=C_{3,(0)} C_{1,(1)} B_{3,(2)} B_{2,(1)} A_{2,(2)}, & \eta^{\prime \prime}{ }_{16}=C_{3,(0)} C_{1,(0)} B_{3,(1)} B_{2,(2)} A_{1,(2)}, \\
\eta^{\prime \prime}{ }_{17}=C_{3,(0)} C_{1,(0)} B_{3,(2)} B_{2,(2)} A_{1,(2)}, & \eta^{\prime \prime}{ }_{18}=C_{3,(0)} C_{1,(0)} B_{3,(1)} B_{2,(0)} A_{2,(1)} A_{1,(2)}, \\
\eta^{\prime \prime}{ }_{19}=C_{3,(0)} C_{1,(0)} B_{3,(1)} B_{2,(0)} A_{2,(2)} A_{1,(2)}, & \eta^{\prime \prime}{ }_{20}=C_{3,(0)} C_{1,(0)} B_{3,(1)} B_{2,(1)} A_{2,(1)} A_{1,(2)}, \\
\eta^{\prime \prime}{ }_{21}=C_{3,(0)} C_{1,(0)} B_{3,(1)} B_{2,(0)} A_{2,(2)} A_{1,(2)}, & \eta^{\prime \prime}{ }_{22}=C_{3,(0)} C_{1,(0)} B_{3,(2)} B_{2,(0)} A_{2,(1)} A_{1,(2)}, \\
\eta^{\prime \prime}{ }_{13}=C_{3,(0)} C_{1,(0)} B_{3,(2)} B_{2,(0)} A_{2,(2)} A_{1,(2)}, & \eta^{\prime \prime}{ }_{24}=C_{3,(0)} C_{1,(0)} B_{3,(2)} B_{2,(1)} A_{2,(1)} A_{1,(2)}, \\
\eta^{\prime \prime}{ }_{25}=C_{3,(0)} C_{1,(0)} B_{3,(2)} B_{2,(1)} A_{2,(2)} A_{1,(2)}, &
\end{array}
$$




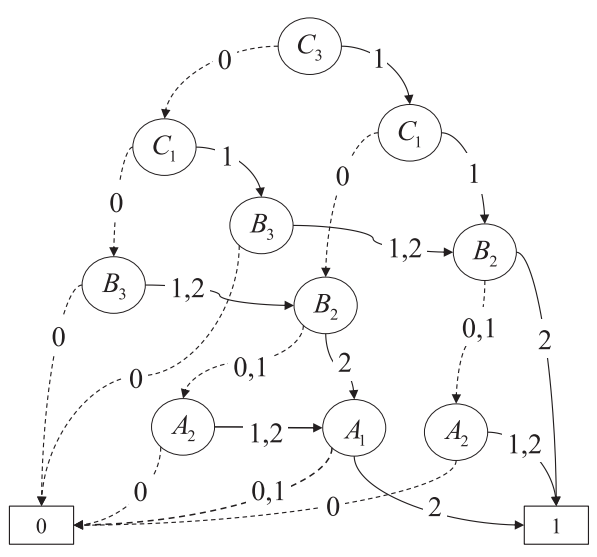

Fig. 9. The system MMDD model for the example PMS.

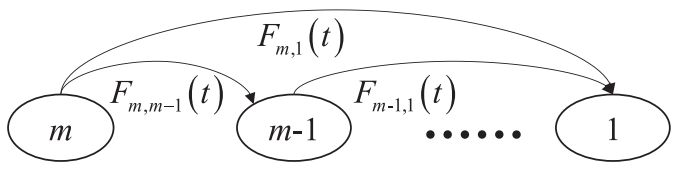

Fig. 10. Transition diagram for the Semi-Markov Process.

Step 4. Considering the characteristic in the non-repairable PMS that the component state in the latter phase cannot be better than that in the former phases, cancel the self-conflict paths in Eq. (12). For example, in $\eta^{\prime \prime}{ }_{8}=C_{3,(0)} C_{1,(1)} B_{3,(1)} B_{2,(0)} A_{2,(1)}, B_{2,(0)}$ and $B_{3,(1)}$ cannot exist in the same path because the components cannot be repaired.

Compared to the general MMDD method, the proposed PMS-MMDD model can do the cancelation of the self-conflict paths in the model construction process, which can make the modelling process more efficient.

In synthesis, the phase algebra for the multi-state PMS and PMS-MMDD model have been proposed to model the multistate PMS consisting of non-repairable multi-state components in this section. Compared to the PMS-BDD method and general MMDD model, the PMS-MMDD model can do the system modelling process with less nodes and the self-conflicting paths can be automatically cancelled and the system MMDD model can be generated without additional steps.

\section{Model evaluation and validation}

The exponential distributions are used in many existing works on the reliability analysis of the PMSs. In the reliability assessment of the multi-state PMS, with the exponential transition time distributions, the path probabilities can be evaluated by the Continuous Time Markov Chain due to its memoryless property [5], the path probability can be evaluated by multiply the components' state probabilities phase by phase. However in the practical applications, most of the components or systems follow the non-exponential distributions, such as the Weibull distribution [28,31]. With the non-exponential distributions, the path evaluation method based on the CTMC is not available. So based on Semi-Markov process [32], a Markov renewal equation-based method is developed to evaluate the path probabilities in this paper. Then, a numerical method is applied to compute the complicated integrals and provide an accurate approximation solution.

\subsection{The Semi-Markov process}

A continuous-time stochastic process is called a Semi-Markov Process (SMP) if the embedded chain is a Markov chain and the transition times between two states follow arbitrary distributions [33,34]. The SMP is a generalization of the classical Markov chain and it accommodates arbitrary transition time distributions. The SMP does not possess the Markov property only except for transition time points, called Markov regeneration epochs because the non-exponential distributions do no possess the non-memoryless property. A SMP model for a non-repairable multi-state system with $m$ states is shown in Fig. 10 .

To evaluate the system state probabilities at time $t$, two matrices, the matrix $\boldsymbol{\theta}(t)$ and the matrix $\boldsymbol{Q}(t)$, are necessary. In matrix $\boldsymbol{\theta}(t)$, each element $\theta_{i, j}(t), i, j \in\{1,2, \ldots, M\}$ represents the probability that the process goes from state $i$ to state $j$ in the time interval $[0, t]$. The matrix $\boldsymbol{Q}(t)$ is known as the kernel matrix, whose element $Q_{i, j}(t),(i \neq j \in\{1,2, \ldots, M\})$ denotes the probability of transition from state $i$ to state $j$ during the time interval $[0, t]$ with one transition step. The kernel matrix $\boldsymbol{Q}(t)$ can be obtained from the cumulative distribution function (CDF) of sojourn times and the competition behaviors among transitions from one specific state. 


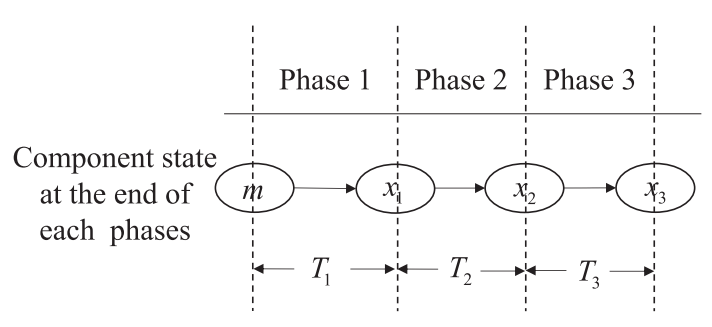

Fig. 11. Example path for component $S$.

With the kernel matrix $\boldsymbol{Q}(t)$, the state probabilities $\theta_{i, j}(t), i, j=\{1,2, \ldots, m\}$ can be derived by solving the integrals given below [3],

$$
\theta_{i, j}(t)=\sigma_{i, j}\left(1-F_{i}(t)\right)+\sum_{k=1}^{m} \int_{0}^{t} q_{i, k}(\tau) \theta_{k, j}(t-\tau) d \tau
$$

where, $q_{i, k}(t)=\frac{d Q_{i, k}(t)}{d t}, F_{i}(t)=\sum_{j=1}^{K} Q_{i, j}(t)$ and $\sigma_{i, j}=\left\{\begin{array}{lll}1, & \text { if } & i=j \\ 0, & \text { if } & i \neq j\end{array}\right.$

Although SMP can deal with non-exponential distributions by solving the Markov renewal equation in Eq. (13), the integrals are difficult to solve analytically with the non-exponential distributions (e.g., Weibull distribution). To solve the complex integral equations, a two-point trapezoidal rule [12] is applied, shown as,

$$
\int_{0}^{t} q_{i, k}(\tau) \theta_{k, j}(t-\tau) d \tau=\frac{1}{2} \sum_{i=0}^{n-1}\left[q_{i, k}\left(\tau_{i}\right) \theta_{k, j}\left(t-\tau_{i}\right)+q_{i, k}\left(\tau_{i+1}\right) \theta_{k, j}\left(t-\tau_{i+1}\right)\right]\left[\tau_{i+1}-\tau_{i}\right]
$$

where the integration interval $[0, t]$ is divided into $n$ equal segments, so the length of each segment is $\delta=t / n$, and the discrete points are $0=\tau_{0} \leq \tau_{1} \leq \cdots \leq \tau_{n-1} \leq \tau_{n}=t$.

With Eq. (14), the Markov renewal equations in Eq. (13) can be expressed as,

$$
\theta_{i, j}(t) \approx \sigma_{i, j}\left(1-F_{i}(t)\right)+\frac{1}{2} \sum_{k=1}^{m} \sum_{i=0}^{n-1}\left[q_{i, k}\left(\tau_{i}\right) \theta_{k, j}\left(t-\tau_{i}\right)+q_{i, k}\left(\tau_{i+1}\right) \theta_{k, j}\left(t-\tau_{i+1}\right)\right]\left(\tau_{i+1}-\tau_{i}\right)
$$

By Eq. (15), all the system states probabilities $\theta_{i, j}(t)$ can be evaluated at any time $t$, recursively.

\subsection{The Markov-renewal equation based path evaluation method}

As the multi-state components are non-repairable and all the components are independent on each other in this paper, so the path probability can be evaluated by multiplying all the components multi-state probabilities in the different phases. For example, the probability of path $\eta_{6}$ in the example PMS can be evaluated as,

$$
\begin{aligned}
\operatorname{Pr}\left\{\eta_{6}=1\right\} & =\operatorname{Pr}\left(C_{3,(0)} C_{1,(1)} B_{3,(1)} B_{2,(2)}\right) \\
& =\operatorname{Pr}\left(C_{1,(1)} C_{3,(0)}\right) \operatorname{Pr}\left(B_{2,(2)} B_{3,(1)}\right)
\end{aligned}
$$

The non-exponential state transition time distributions are studied in this paper and they do not possess the memoryless property, such as the Weibull distribution. With the SMP as well the Markov renewal equation, a Markov-renewal equationbased method is proposed to evaluate the path probabilities with non-exponential transition time distributions.

To show the Markov renewal equation-based method, component $S$ with $m$ states ( $m$ is the best state and 1 is the worst state) and its multi-state behaviors in three phases are shown in Fig. 11, in which $x_{1}, x_{2}$ and $x_{3}\left(x_{1}, x_{2}, x_{3} \in[m, m-1, \ldots\right.$, 1] and $m \geq x_{1} \geq x_{2} \geq x_{3}$ ) represent the state of component $S$ at the end of phases 1,2 and 3, respectively.

As we all know, the transitions between two states (the states at the beginning and the end of one phase) can occur at any time during the phase, for example, component $\mathrm{A}$ can transit from state $m$ to state $x_{1}$ at any time in phase 1 . Using $\tau_{1}$, $\tau_{2}$ and $\tau_{3}$ to represent the specific time of component $\mathrm{A}$ arriving state $x_{1}, x_{2}$ and $x_{3}$, the path can be divided into several time intervals according to the component state at different time, shown in Fig. 12.

From the Fig. 12, we can get the integral interval length of $\tau_{1}, \tau_{2}$ and $\tau_{3}$ are $\left[0, T_{1}\right],\left[0, T_{2}\right]$ and $\left[0, T_{3}\right]$, respectively. Then the path probability can be evaluated by,

$$
\begin{aligned}
\operatorname{Pr} & \left(S_{0}=m, S_{T_{1}}=x_{1}, S_{T_{2}}=x_{2}, S_{T_{3}}=x_{3}\right) \\
& =\int_{0}^{T_{1}} f_{m, x_{1}}\left(\tau_{1}\right) \int_{0}^{T_{2}} f_{x_{1}, x_{2}}\left(\tau_{2}+T_{1}-\tau_{1}\right) \int_{0}^{T_{3}} f_{x_{2}, x_{3}}\left(\tau_{3}+T_{2}-\tau_{2}\right) \theta_{x_{3}, x_{3}}\left(T_{3}-\tau_{3}\right) d \tau_{3} d \tau_{2} d \tau_{1}
\end{aligned}
$$

where the $f_{i, j}(\tau), i \geq j$ represent the probability density function (pdf) that component $A$ transit from state $i$ to state $j$. And the last transition to state $j$ occurs at time $\tau$. All the $f_{i, j}(\tau)$ can be divided into three categories according to the relationship between $i$ and $j$, 


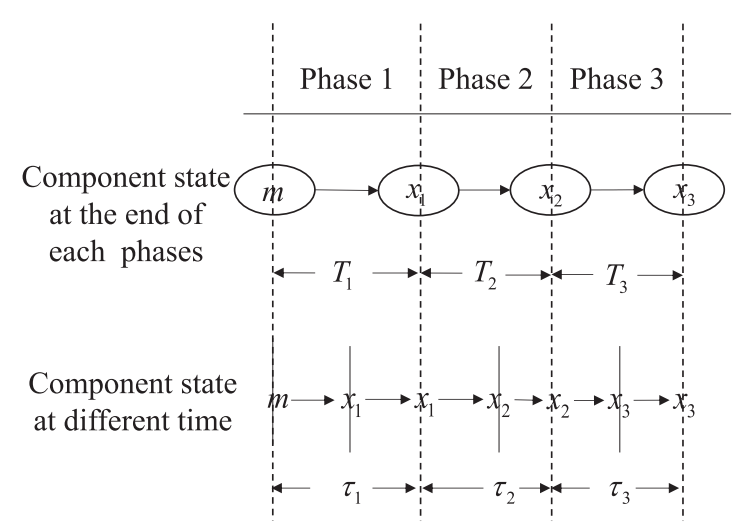

Fig. 12. Time interval division according to component state.

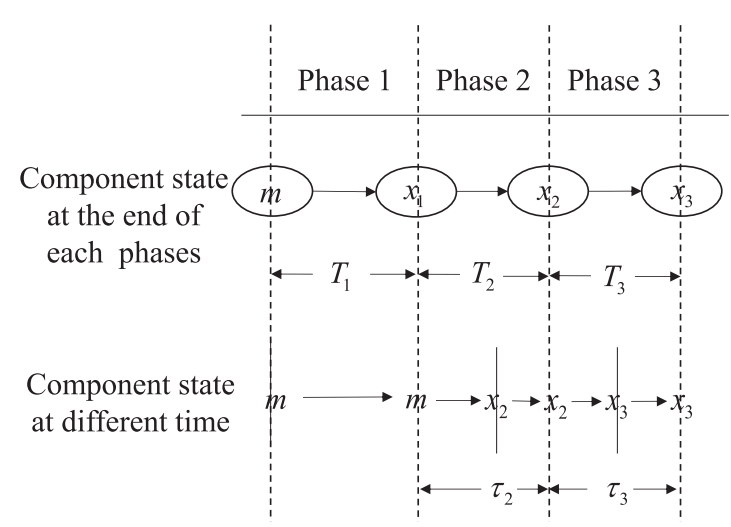

Fig. 13. Time interval division according to component state if $m=x_{1}$.

(1) $i=j$, which means the component stays in one specific state and no transition occurs in this phase. In this case, the integral for this phase can be combined into next phase. For example, if $x_{1}=m$ in Eq. (17), the time interval division can be changed as shown in Fig. 13.

Then Eq. (17) can be derived as,

$$
\begin{aligned}
\operatorname{Pr} & \left(S_{0}=m, S_{T_{1}}=m, S_{T_{2}}=x_{2}, S_{T_{3}}=x_{3}\right) \\
& =\int_{0}^{T_{2}} f_{m, x_{2}}\left(\tau_{2}+T_{1}\right) \int_{0}^{T_{3}} f_{x_{2}, x_{3}}\left(\tau_{3}+T_{2}-\tau_{2}\right) \theta_{x_{3}, x_{3}}\left(T_{3}-\tau_{3}\right) d \tau_{3} d \tau_{2}
\end{aligned}
$$

(2) $i-j=1$, which means only one transition occurs in this phase. Therefore, the $f_{i, j}(\tau)=q_{i, j}(\tau)=\frac{d Q_{i, k}(t)}{d t}$.

(3) $i-j>1$, which means more than one transition occur in this phase. To evaluate the $f_{i, j}(t)$, a matrix $\mathbf{K}(t)$ for the SemiMarkov process with $m$ states is proposed in this paper. And the element in $K(t), K_{i, j}(t)$, means that the process transit from state $i$ to state $j$ in the time interval $[0, t]$ with finite steps and the last transition to state $j$ occurs at time $t$. Then, $K_{i, j}(t)$ can be evaluated by,

$$
K_{i, j}(t)=\sum_{k=1}^{m} \int_{0}^{t} q_{i, k}(\tau) K_{k, j}(t-\tau) d \tau
$$

Eq. (19) can be evaluated by the numerical method shown in Eq. (14) recursively. Comparing $\boldsymbol{\theta}(\boldsymbol{t})$ and $\boldsymbol{K}(t)$, we can find the difference is that $\theta_{i, j}(t)$ considers the probability that the process stays in the same state in one period (when $i=j$ ) and $K_{i, j}(t)$ does not. For example, the $\boldsymbol{\theta}(t)$ and $\boldsymbol{K}(t)$ for the SMP in Fig. 10 are given by,

$$
\boldsymbol{\theta}(t)=\left[\begin{array}{cccc}
\theta_{m, m}(t) & \theta_{m, m-1}(t) & \cdots & \theta_{m, 1}(t) \\
0 & \theta_{m-1, m-1}(t) & \cdots & \theta_{m-1,1}(t) \\
0 & 0 & \cdots & \cdots \\
0 & 0 & \cdots & \theta_{1,1}(t)
\end{array}\right]
$$


Not invisible: (1) Computers and software(A)

(2) Battery(B)

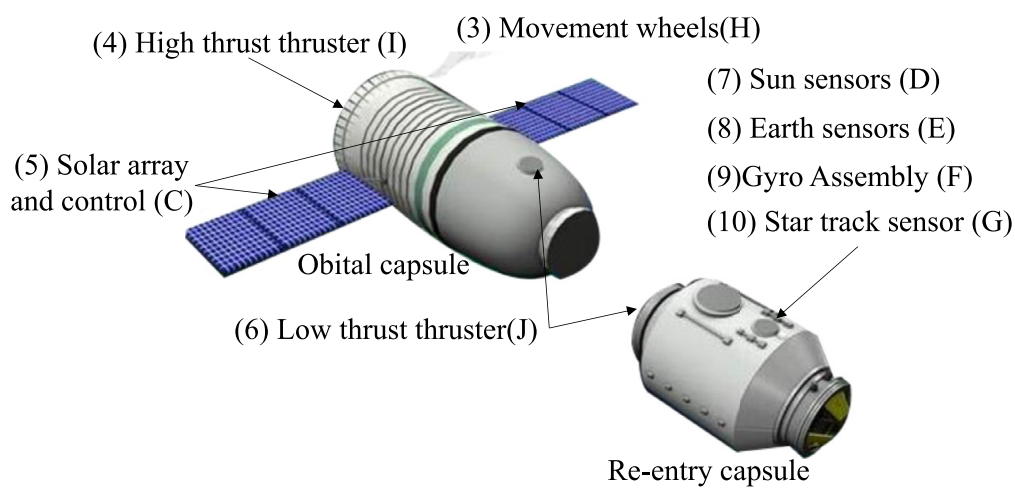

Fig. 14. The spacecraft and the functional components of its AOCS.

$$
\boldsymbol{K}(t)=\left[\begin{array}{ccccc}
0 & K_{m, m-1}(t) & K_{m, m-2}(t) & \cdots & K_{m, 1}(t) \\
0 & 0 & K_{m-1, m-2}(t) & \cdots & K_{m-1,1}(t) \\
0 & 0 & 0 & \cdots & K_{m-2,1}(t) \\
0 & 0 & 0 & \cdots & \cdots \\
0 & 0 & 0 & \cdots & 0
\end{array}\right]
$$

Then, with the evaluated $\boldsymbol{K}(t)$, if $m-x_{1}>1$, Eq. (17) can be derived as,

$$
\begin{aligned}
\operatorname{Pr} & \left(S_{0}=m, S_{T_{1}}=m, S_{T_{2}}=x_{2}, S_{T_{3}}=x_{3}\right) \\
& =\int_{0}^{T_{1}}\left(\sum_{k=1}^{m} \int_{0}^{\tau_{1}} q_{i, k}\left(\tau_{1}^{\prime}\right) K_{k, j}\left(t-\tau_{1}^{\prime}\right) d \tau_{1}^{\prime}\right) \int_{0}^{T_{2}} f_{x_{1}, x_{2}}\left(\tau_{2}+T_{1}-\tau_{1}\right) \\
& \int_{0}^{T_{3}} f_{x_{2}, x_{3}}\left(\tau_{3}+T_{2}-\tau_{2}\right) \theta_{x_{3}, x_{3}}\left(T_{3}-\tau_{3}\right) d \tau_{3} d \tau_{2} d \tau_{1}
\end{aligned}
$$

With the Markov renewal equation-based method, the path probabilities can be evaluated by the following two steps,

Step 1, use the numerical approximation method and evaluate the state probability matrix $\boldsymbol{\theta}(t)$ and $\boldsymbol{K}(t)$.

Step 2, divide all the phases into several time intervals according to the components' states in different phases. Then, construct the multi-integral function for path probability evaluation by the Markov renewal equation-based method and ascertain all the $f_{i, j}(\tau)$. Then, evaluate multi-integrals by the numerical algorithm [35] recursively from the inner integral to the outer integral step by step.

\section{Case study and valiation}

\subsection{Case study}

\subsubsection{System structure and multi-state components}

In this section, an attitude and orbit control system (AOCS) from a real engineering project is used to illustrate the proposed PMS-MMDD model and the Markov renewal equation-based method. The AOCS is a critical subsystem that is used to keep the spacecraft in the correct attitude and orbit in its whole lifetime. There are 10 functional components in the AOCS of the spacecraft, as shown in Fig. 14.

All the components can be divided into four groups according to their own functions: (1) Electric power subsystem, includes the battery (B) and the solar array and control (C). The electric power system is used for providing the electrical power to all other components; (2) sensor subsystem, includes the sun sensor (D), earth sensor (E), Gyro assembly (F) and star track sensor $(G)$. Different sensors are used in different phases to collect the attitude and orbit data; (3) control subsystem, includes the computers and software (A). The control subsystem process the data from sensors and make decisions, then give instructions to the actuators; (4) dynamics subsystem, includes the high thrust thruster (I), low thrust thruster (J) and the movement wheels $(\mathrm{H})$. According to the instructions from the control subsystem, the dynamics subsystem adjusts the attitude and orbit. The working procedure of the AOCS is shown in Fig. 15. The repeating of this working procedure keeps the spacecraft in correct attitude and orbit. 


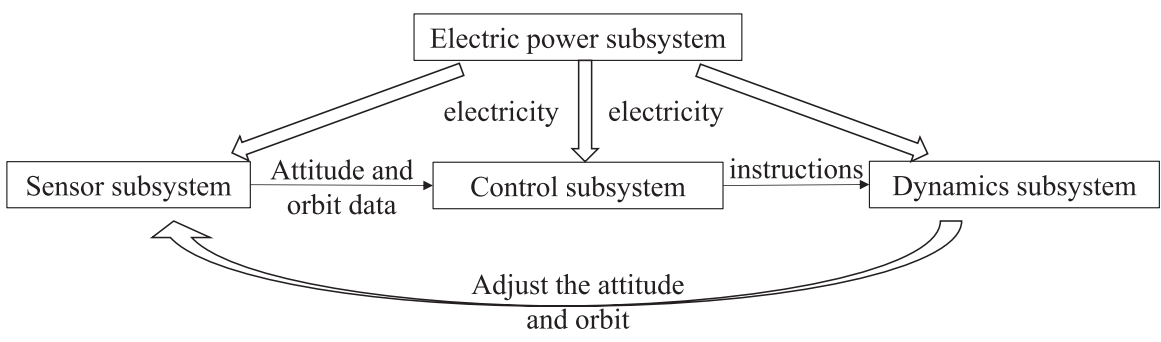

Fig. 15. The working procedure of the AOCS in spacecraft.

Table III

The parameters for the components in the AOCS

\begin{tabular}{llllllll}
\hline & $\mathrm{A}$ & $\mathrm{B}$ & $\mathrm{C}$ & $\mathrm{D} / \mathrm{E} / \mathrm{F} / \mathrm{G}$ & $\mathrm{H}$ & $\mathrm{I}$ & $\mathrm{J}$ \\
\hline$\alpha_{3,2}$ & 1.6093 & 2.4136 & 2.9417 & 1.9284 & 0.8826 & 3.9359 & 4.3064 \\
$\beta_{3,2}$ (days) & 1067.08 & 856.76 & 2861.76 & 3480.29 & 3658.66 & 2263.81 & 2209.64 \\
$\alpha_{2,1}$ & 1.2570 & 1.2722 & 3.3196 & 2.7396 & 3.1182 & 0.7533 & 1.7094 \\
$\beta_{2,1}$ (days) & 1955.75 & 1852.89 & 1732.87 & 791.38 & 2.7733 & 2149.07 & 1604.77 \\
\hline
\end{tabular}

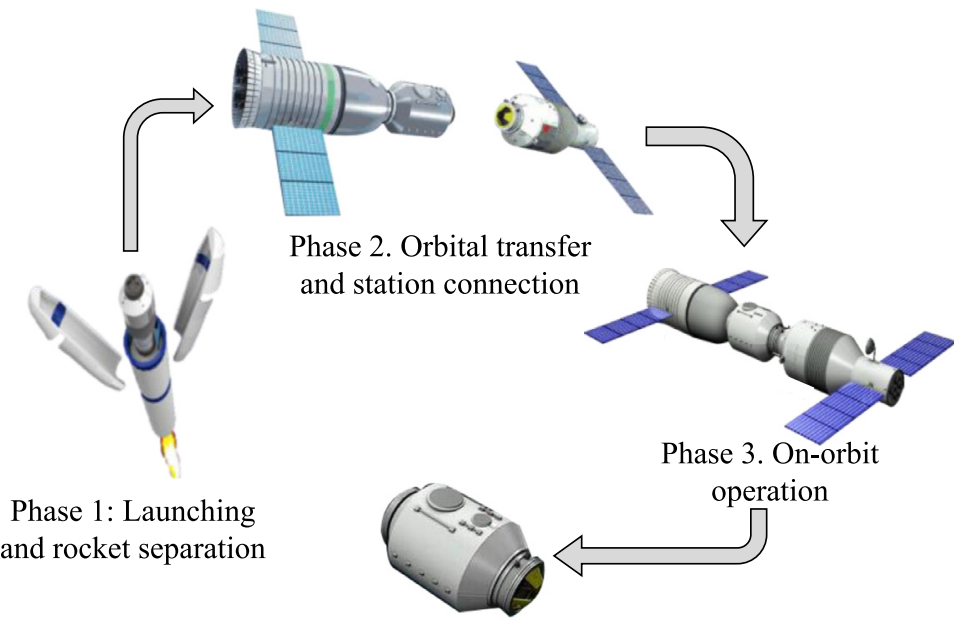

Phase 4. Back to earth

Fig. 16. The mission profile for the AOCS of the spacecraft.

All the components in the AOCS have three states according to their own characteristics: (1) State 2, fully operational state. The performance of the component is in the best state. For example, majority of the solar cell is good and can provide full power or both the redundancy can work normally; (2) state 1, degradation state or partial failure state. The components only have partial performance, like the solar array, only part of the solar cell works well and can only provide partial power. (3) state 0 , complete failure state.

Due to the confidentiality of the aerospace project, the failure time data is not available. All the components' state transition distributions and the parameters are provided by the aerospace designers and labelled in Table III. All the parameters are estimated by the data from harsh experiments to ensure the safety of the spacecraft. The state transition distributions follow the Weibull distribution $\left(F(t)=1-e^{\left(-(t / \alpha)^{\beta}\right)}\right)$ in which $\alpha$ and $\beta$ are the shape parameter and scale parameter, respectively. All the $\beta$ values are given in days.

\subsubsection{Fault tree model of each phase}

According to the different missions in different phases, the lifetime of the AOCS in the spacecraft can be divided into four phases, as shown in Fig. 16.

In phase 1 , the whole spacecraft is launched to the outer space and separated from the rocket. Then, the solar array is unfolded correctly. The phase duration is $T_{1}=1$ day. In this phase, the AOCS needs to adjust the orbit and ensure the successful stretching of solar array. Component A needs to be in state 2 or state 1 and component B, C, E, and G need to be in state 2. The phase fault tree model is shown as Fig. 17(a). 


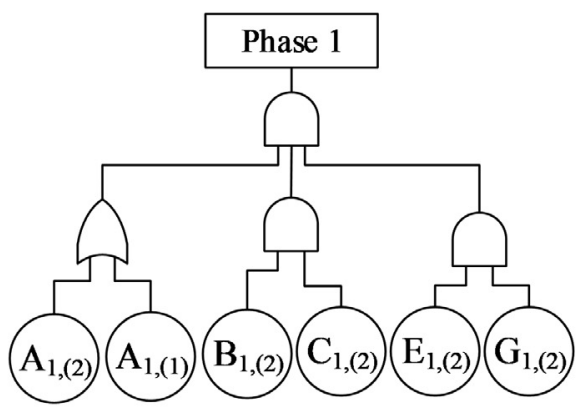

(a)

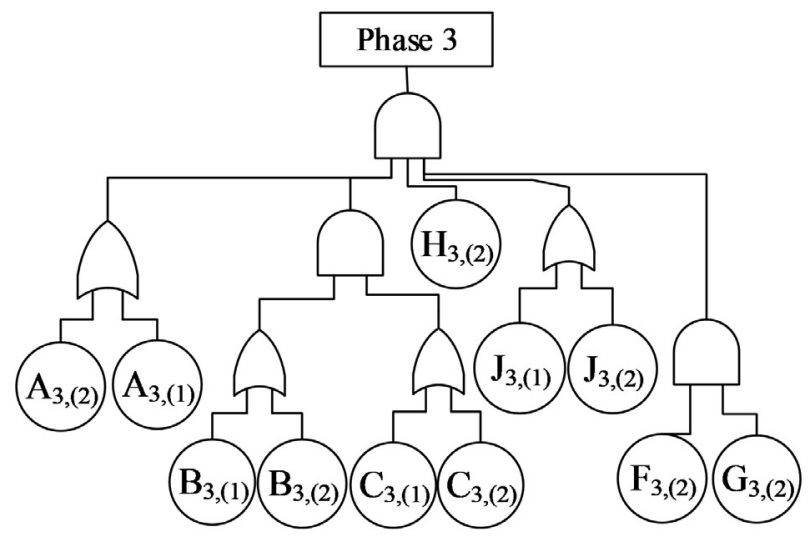

(c)

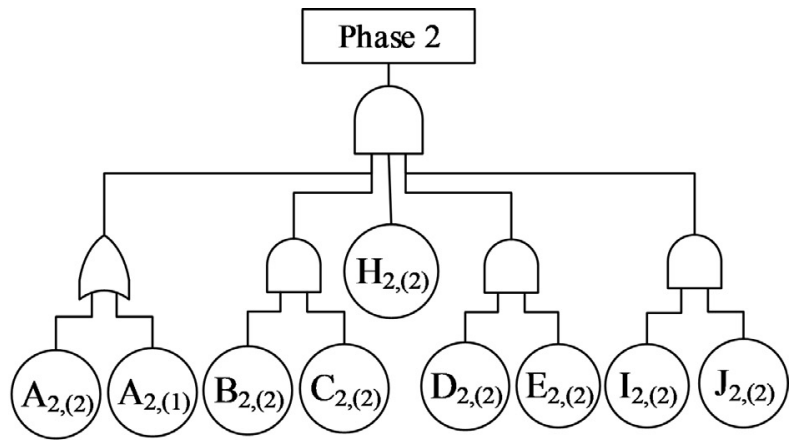

(b)

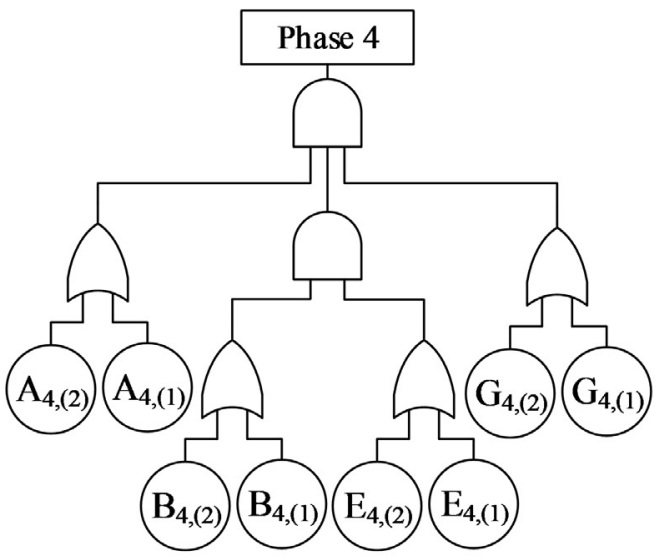

(d)

Fig. 17. The fault tree model for each phase of the AOCS.

In phase 2, the spacecraft is transferred to the working orbit and connected to the space station. In this phase, the AOCS needs to provide full power to transfer to the orbit until the whole spacecraft is in the working orbit accurately and connect to the space station. The phase duration is $T_{2}=3$ days. Component A needs to be in state 2 or state 1 and component $\mathrm{B}, \mathrm{C}$, D, E, I, J and $\mathrm{H}$ need to be in state 2. The phase fault tree model is shown as Fig. 17(b).

In phase 3 , the spacecraft works in the working orbit with the space station for nearly one month and the astronauts need to finish the tasks. The AOCS needs to acquire highly accurate data and keep the spacecraft in the correct attitude and orbit. The phase duration is $T_{2}=24$ days. Components $\mathrm{A}, \mathrm{B}, \mathrm{C}$ and $\mathrm{J}$ need to be in state 2 or state 1 and components $\mathrm{F}, \mathrm{G}$ and $\mathrm{H}$ need to be in state 2. The phase fault tree model is shown as Fig. 17(c).

In phase 4, part of the spacecraft, the re-entry capsule is separated from the station and sent back to earth with the astronauts. The AOCS needs to separate the re-entry capsule from the station and send it to the low orbit and then send it back to earth safely. The phase duration is $T_{2}=2$ days. Components $\mathrm{A}, \mathrm{B}, \mathrm{E}$ and $\mathrm{J}$ need to be in state 1 or state 2 . The phase fault tree model is shown as Fig. 17(d).

Then, by the proposed PMS-MMDD method proposed in Section 3 and the fault tree model of each phase in Fig. 17, the system MMDD model is generated with the backward PDD, whose ordering of the variables is: $J_{3}<J_{2}<I_{2}<H_{3}<H_{2}<$ $G_{4}<G_{3}<G_{1}<F_{3}<E_{4}<E_{2}<E_{1}<D_{2}<C_{3}<C_{2}<C_{1}<B_{4}<B_{3}<B_{2}<B_{1}<A_{4}<A_{3}<A_{2}<A_{1}$. The system MMDD model for the AOCS is shown as Fig. 18.

Then, there are totally 32 paths from the root node ' $J 3$ ' to the sink node ' 1 ' in Fig. 18 ,

$$
\begin{aligned}
& \eta_{1}^{A O C S}=J_{3,(2)} I_{2,(2)} H_{3,(2)} G_{4,(2)} F_{3,(2)} E_{4,(2)} D_{2,(2)} C_{3,(2)} B_{4,(2)} A_{4,(1,2)} \\
& \eta_{2}^{A O C S}=J_{3,(1)} J_{2,(2)} I_{2,(2)} H_{3,(2)} G_{4,(2)} F_{3,(2)} E_{4,(2)} D_{2,(2)} C_{3,(2)} B_{4,(2)} A_{4,(1,2)} \\
& \ldots \ldots . \\
& \eta_{32}^{A O C S}=J_{3,(1)} J_{2,(2)} I_{2,(2)} H_{3,(2)} G_{4,(1)} G_{3,(2)} F_{3,(2)} E_{4,(1)} E_{2,(2)} D_{2,(2)} C_{3,(1)} C_{2,(2)} B_{4,(1)} B_{2,(2)} A_{4,(1,2)}
\end{aligned}
$$




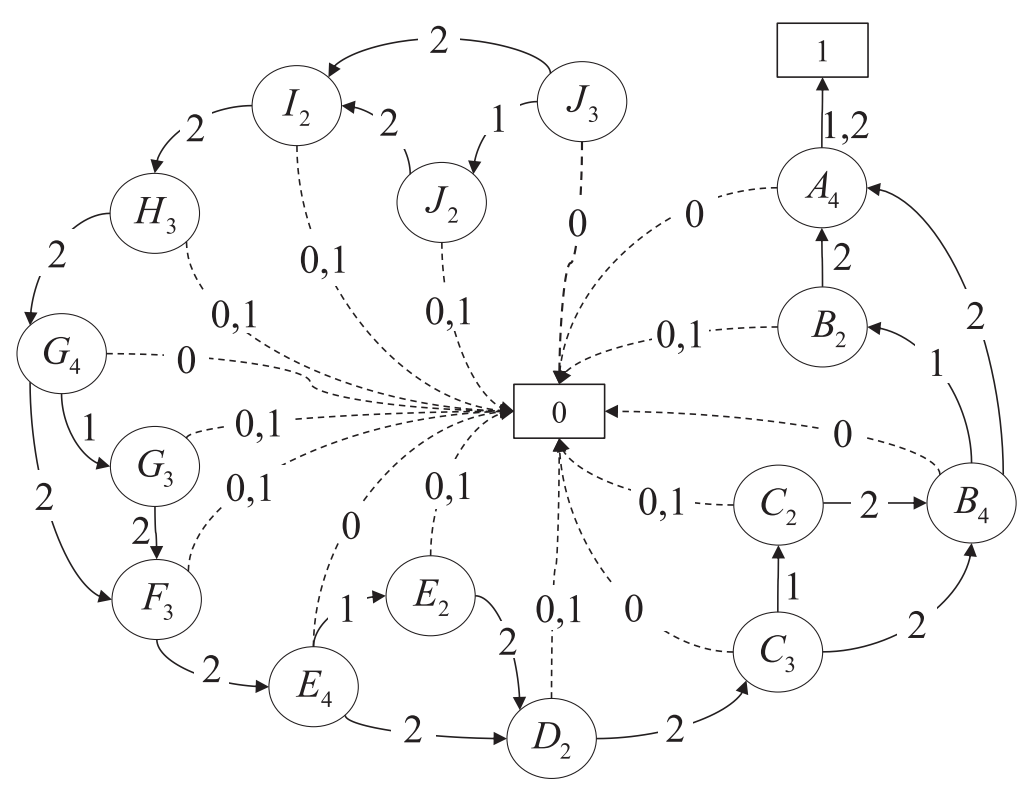

Fig. 18. The system MMDD model of the AOCS.

By the Markov-renewal equation-based method proposed in Section 4 with the length of each segment $\delta=0.1$, the probability of each path can be evaluated as,

$$
\begin{aligned}
& \eta_{1}^{\text {AOCS }}=0.9103 \\
& \eta_{2}^{\text {AOCS }}=1.6413 \times 10^{-4} \\
& \cdots \ldots . \\
& \eta_{32}^{\text {AOCS }}=4.8722 \times 10^{-15}
\end{aligned}
$$

Then, the system reliability at the end of the last phase can be evaluated as,

$$
R_{\text {sys }}^{\text {AOCS }}=\sum_{i=1}^{32} \eta_{i}^{A O C S}=0.93032
$$

\subsection{Validation}

In this section, to demonstrate the effect of the proposed the Markov renewal equation-based path evaluation method, a comparison among the proposed method, the path evaluation method used in [5] and the Monte Carlo simulation method is carried out. Then, the proposed method is compared to the Monte Carlo simulation method under different numbers of realizations to show its accuracy and computation efficiency.

\subsubsection{Comparison to existing model}

By the path evaluation method in [5], the component's state probability at time $t$ in phase $i$ is evaluated by multiplying the component's state probability at the beginning of phase $i$ and its transition probability at time $t$ in this phase. The Monte Carlo simulation method is used for verification, with the simulation procedure shown in Fig. 19. The comparison of the assessed AOCS reliabilities by these three methods are shown in Fig. 20.

From the comparison of the system reliability curves assessed by different models, we can conclude that the proposed method can provide an accurate result, whereas the classical method can lead to overestimating the system reliability in the non-exponential multi-state PMS. The main reason is that in the classical model, the component is regarded as new at the beginning of each phase, which makes the assessed system reliability higher than the true value.

\subsubsection{Accuracy and computation efficiency validation}

In this section, the comparison between the proposed method and the Monte Carlo simulation method is carried out. In this paper, the exact system reliability cannot be assessed analytically, so the system reliability is compared by the Monte Carlo simulation method with $N_{\max }=10^{8}$ realizations are used as a 'true' value [36] for the comparisons.

The accuracy and computation efficiency of the Monte Carlo simulation method are highly dependent on the numbers of realizations [37,38]. The errors between the results of the Monte Carlo simulation method under different numbers of 


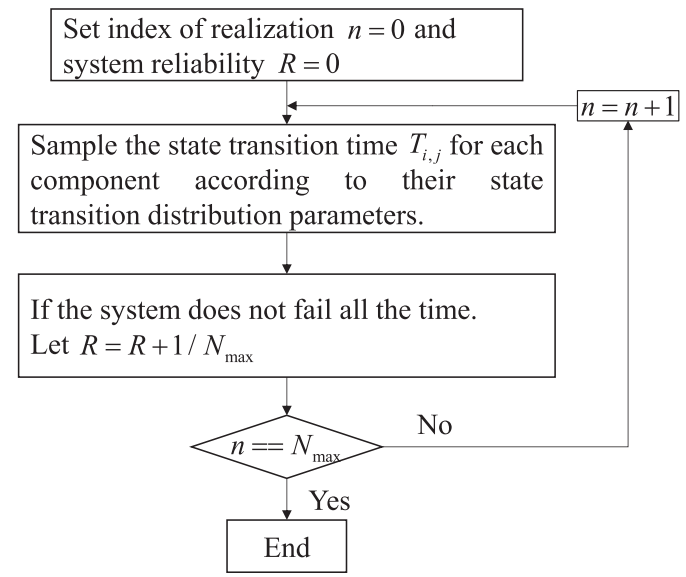

Fig. 19. Monte Carlo simulation procedure.

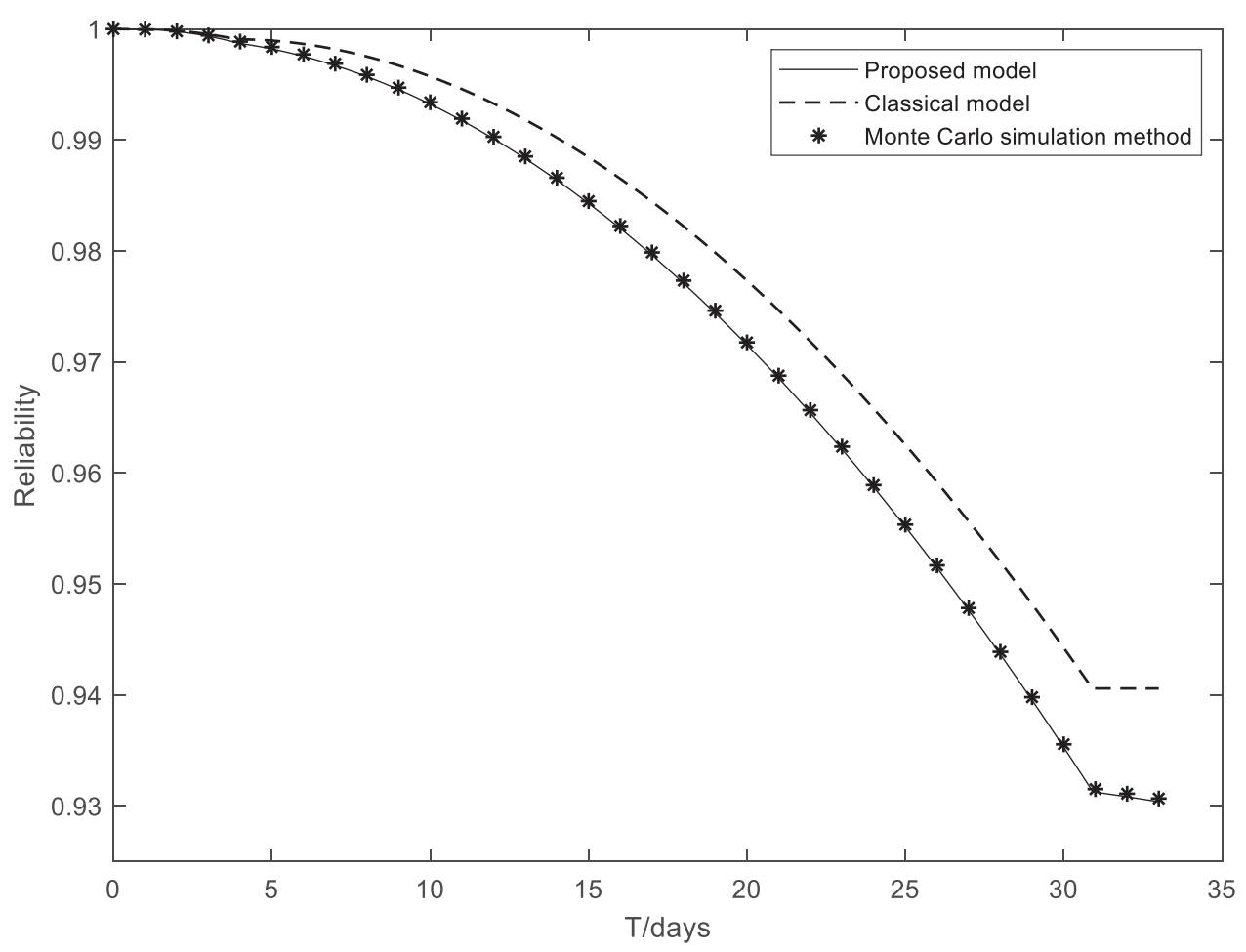

Fig. 20. Comparison of the AOCS reliability under different models.

realizations and the true value are shown in the second row in Table IV. The errors are evaluated as the mean value of 10 repetitions to ensure their stability and the computation time by the Monte Carlo simulation method with different number of realizations are shown in the third row in Table IV.

The accuracy and computation efficiency of the proposed method are highly dependent on the segment length $\delta$ of the numerical method. The computation time and errors of the proposed method with different segment lengths are shown in Table V.

From the errors and calculation time comparison between Tables IV and V, we can see that the proposed method can provide an accurate result with high computational efficiency, compared to the Monte Carlo simulation method. 
Table IV

Computation time and errors of Monte Carlo simulation method.

\begin{tabular}{llllll}
\hline Number of realizations & $2 \times 10^{2}$ & $2 \times 10^{3}$ & $2 \times 10^{4}$ & $2 \times 10^{5}$ & $2 \times 10^{6}$ \\
Errors & 0.0130 & $5.883 \times 10^{-3}$ & $1.167 \times 10^{-3}$ & $6.049 \times 10^{-4}$ & $2.005 \times 10^{-4}$ \\
Computation time(s) & 0.044 & 0.187 & 2.85 & 15.52 & 155.69 \\
\hline
\end{tabular}

Table V

Computation time and errors of the proposed method.

\begin{tabular}{lllll} 
Number of realizations & $\delta=1$ & $\delta=0.5$ & $\delta=0.1$ & $\delta=0.01$ \\
Errors & $1.370 \times 10^{-3}$ & $6.940 \times 10^{-4}$ & $1.517 \times 10^{-4}$ & $2.881 \times 10^{-5}$ \\
Computation time(s) & 0.061 & 0.071 & 0.141 & 1.302 \\
\hline
\end{tabular}

\section{Conclusions and future works}

In this paper, a PMS-MMDD method is proposed to model a multi-state PMS consisting of non-repairable multi-state components. Compared to the existing PMS-BDD method, the scale of the PMS-MMDD system model is smaller. Moreover, compared to the general MMDD method, the dependency among the phases can be fully considered in the model generation process and the conflicting paths can be automatically cancelled without any additional analysis step by the proposed PMSMMDD method, which makes the reliability modelling simpler. Then, to deal with non-exponential multi-state components, a Markov renewal equation-based method is developed to evaluate the probabilities of the paths generated by the PMSMMDD method. By the Markov renewal equation-based path evaluation method, the time-dependent failure rates of nonexponential distributions can be considered across phases and then, the system reliability can be assessed by a numerical method.

The applicability of proposed method has been demonstrated on a real engineering system, i.e., the attitude and orbit control system in a spacecraft. From the example, we can see that the system reliability at any time can be assessed efficiently by the proposed method. The traditional path evaluation method, which is based on the assumption that all components are regarded as new at the beginning of each phase, results in an overestimated system reliability for the non-exponential PMS. Compared to the Monte Carlo simulation method, the proposed method can provide similar accurate results but with a much higher computation efficiency. As the system reliability can be assessed fast and accurately, the proposed method can be further utilized for the reliability optimization allocation problem and evaluation of other reliability indices of the multi-state PMS.

In this paper, the MMDD models are generated manually by phase algebra for MMDD which can become complicated in the modelling of large scale systems. Therefore, implementing the proposed method in software is part of our future work. After, the relationship between the system scale and the variable orders will be further studied in the multi-state PMS software programming. On the other hand, the system structure may be more complex and dynamic, such as cold standby or functional standby competition: how to model and assess these dynamic behaviors in the multi-state PMS will be another topic of future works.

\section{Acknowledgement}

This study was sponsored by the National Natural Science Foundation of China under Grant No. 51775090 and China Scholarship Council No. 201606070066.

\section{Appendix}

\section{A1. Proof of Eq. (8)}

If component A stays in one specific state in phase $i$, in the case of no-repairs, its state in phase $j$ should not be better than its state in phase $i$. so,

$$
\begin{aligned}
& A_{i, m}=1 \rightarrow A_{j, m}=A_{j, m}, A_{j, m-1}=A_{j, m-1}, \ldots, A_{j, 1}=A_{j, 1} \\
& A_{i, m-1}=1 \rightarrow A_{j, m}=0, A_{j, m-1}=A_{j, m-1}, A_{j, m-2}=A_{j, m-2}, \ldots, A_{j, 1}=A_{j, 1} \\
& \ldots \ldots \\
& A_{i, 1}=1 \rightarrow A_{j, m}=0, A_{j, m-1}=0, \ldots, A_{j, 2}=0, A_{j, 1}=1
\end{aligned}
$$

Then,

$$
\begin{aligned}
& \operatorname{case}\left(A_{i}, G_{m}, \ldots, G_{1}\right) \operatorname{case}\left(A_{j}, H_{m}, \ldots, H_{1}\right) \\
& =\operatorname{case}\left(A_{i},\left(E_{i} \diamond E_{j}\right)_{A_{i, m}=1},\left(E_{i} \diamond E_{j}\right)_{A_{i, m-1}=1}, \ldots,\left(E_{i} \diamond E_{j}\right)_{A_{i, 2}=1},\left(E_{i} \diamond E_{j}\right)_{A_{i, 1}=1}\right) \\
& =\operatorname{case}\left(A_{i},\left(E_{i}\right)_{A_{i, m}=1} \diamond\left(E_{j}\right)_{A_{i, m}=1},\left(E_{i}\right)_{A_{i, m-1}=1} \diamond\left(E_{j}\right)_{A_{i, m-1}=1}, \ldots,\left(E_{i}\right)_{A_{i, 2}=1} \diamond\left(E_{j}\right)_{A_{i, 2}=1},\left(E_{i}\right)_{A_{i, 1}=1} \diamond\left(E_{j}\right)_{A_{i, 1}=1}\right)
\end{aligned}
$$




$$
\begin{aligned}
& =\operatorname{case}\left(A_{i}, G_{m} \diamond E_{j, 1}, G_{m-1} \downarrow E_{j, 2}, \ldots, G_{2} \downarrow E_{j, m-1}, G_{1} \downarrow E_{j, m}\right) \\
& \operatorname{case}\left(A_{i}, G_{m} \downarrow E_{j, 1}, G_{m-1} \downarrow E_{j, 2}, \ldots, G_{2} \downarrow E_{j, m-1}, G_{1} \downarrow E_{j, m}\right)
\end{aligned}
$$

and $E_{j, n}=\operatorname{case}(A_{j}, \underbrace{0, \ldots, 0}_{n-1}, H_{m-n+1}, \ldots, H_{2}, H_{1}), 1 \leq n \leq m$, in which, $E_{j, m}=\operatorname{case}(A_{j}, \underbrace{0, \ldots, 0}_{m-1}, H_{1})=H_{1}, E_{j, 1}=\left(A_{j}, H_{m}, \ldots\right.$, $\left.H_{1}\right)=E_{j}$

A2. Proof of Eq. (9)

If component A stays in one specific state in phase $j$, in the case of no repairs, its state in phase $i$ should not be worse than its state in phase $j$. so,

$$
\begin{array}{ll}
A_{j, m}=1 & \rightarrow A_{i, m}=1 \quad A_{i, m-1}=0, A_{i, m-2}=0, \ldots, A_{i, 1}=0 \\
A_{j, m-1}=1 & \rightarrow A_{i, m}=A_{i, m}, A_{i, m-1}=A_{i, m-1}, A_{i, m-2}=0, \ldots, A_{i, 1}=0 \\
\cdots \cdots & \\
A_{j, 1}=1 & \rightarrow A_{i, m}=A_{i, m}, A_{i, m-1}=A_{i, m-1}, \ldots, A_{i, 2}=A_{i, 2}, A_{i, 1}=A_{i, 1}
\end{array}
$$

Hence,

$$
\begin{aligned}
& \operatorname{case}\left(A_{i}, G_{m}, \ldots, G_{1}\right) \operatorname{case}\left(A_{j}, H_{m}, \ldots, H_{1}\right) \\
& =\operatorname{case}\left(A_{j},\left(E_{i} \diamond E_{j}\right)_{A_{j, m}=1},\left(E_{i} \diamond E_{j}\right)_{A_{j, m-1}=1}, \ldots,\left(E_{i} \diamond E_{j}\right)_{A_{j, 2}=1},\left(E_{i} \diamond E_{j}\right)_{A_{j, 1}=1}\right) \\
& =\operatorname{case}\left(A_{j},\left(E_{i}\right)_{A_{i, m}=1} \diamond\left(E_{j}\right)_{A_{i, m}=1},\left(E_{i}\right)_{A_{i, m-1}=1} \diamond\left(E_{j}\right)_{A_{i, m-1}=1}, \ldots,\left(E_{i}\right)_{A_{j, 2}=1} \diamond\left(E_{j}\right)_{A_{i, 2}=1},\left(E_{i}\right)_{A_{i, 1}=1} \diamond\left(E_{j}\right)_{A_{i, 1}=1}\right) \\
& =\operatorname{case}\left(A_{j}, E_{i, 1} \diamond H_{m}, E_{i, 2} \diamond H_{m-1}, \ldots, E_{i, m} \diamond H_{1}\right)
\end{aligned}
$$

and $\quad E_{i, n}=\operatorname{case}(A_{i}, G_{m}, G_{m-1}, \ldots, G_{m-n+1}, \underbrace{0, \ldots, 0}_{m-n}), 1 \leq n \leq m, \quad$ in $\quad$ which $\quad E_{i, 1}=\operatorname{case}(A_{i}, G_{m}, \underbrace{0, \ldots, 0}_{m-1})=G_{m} \quad$ and $E_{i, m}=\operatorname{case}\left(A_{i}, G_{m}, \ldots, G_{1}\right)=E_{i}$.

\section{Reference}

[1] L. Xing, S.V. Amari, Reliability of Phased-Mission systems. Handbook of Performability Engineering, Springer, London, 2008.

[2] L. Xing, Reliability evaluation of phased-mission systems with imperfect fault coverage and common-cause failures, IEEE Trans. Reliab. 56 (2007) $58-68$.

[3] A. Lisnianski, G. Levitin, Multi-state System Reliability: Assessment, Optimization and Applications, World Scientific Publishing Co Inc, Singapore, 2003.

[4] Y.H. Lin, Y.F. Li, E. Zio, Integrating random shocks into multi-state physics models of degradation processes for component reliability assessment, IEEE Trans. Reliab. 64 (2015) 154-166.

[5] A. Shrestha, L. Xing, Y. Dai, Reliability analysis of multistate phased-mission systems with unordered and ordered states, IEEE. Trans. Syst. Man Cybern. A. 41 (2011) 625-636.

[6] Y. Mo, L. Xing, J.B. Dugan, MDD-based method for efficient analysis on phased-mission systems with multimode failures, IEEE Trans. Syst. Man. Cyber. A. 44 (2014) 757-769.

[7] L. Xing, G. Levitin, BDD-based reliability evaluation of phased-mission systems with internal/external common-cause failures, Reliab. Eng. Syst. Safe. 112 (2013) 145-153.

[8] X. Zang, N. Sun, K.S. Trivedi, A BDD-based algorithm for reliability analysis of phased-mission systems, IEEE Trans. Reliab. 48 (1999) 50-60.

[9] L. Xing, J.B. Dugan, Analysis of generalized phased-mission system reliability, performance, and sensitivity, IEEE Trans. Reliab. 51 (2002) 199-211.

[10] M. Librizzi, E. Zio, Direct Monte Carlo simulation for the reliability assessment of a space propulsion system phased mission, in: Proceedings of the Eighth International Conference on Probabilistic Safety Assessment \& Management (PSAM), 2006, pp. 1-9.

[11] F. Tillman, C. Lie, C. Hwang, Simulation model of mission effectiveness for military systems, IEEE Trans. Reliab. 27 (1978) $191-194$.

[12] X. Wu, H. Yan, L. Li, Numerical method for reliability analysis of phased-mission system using Markov chains, Commun. Stat. Theory Methods 41 (2012) 3960-3973.

[13] Z. Tang, J.B. Dugan, BDD-based reliability analysis of phased-mission systems with multimode failures, IEEE Trans. Reliab. 55 (2006) 350-360.

[14] X. Zang, D. Wang, H. Sun, K.S. Trivedi, A BDD-based algorithm for analysis of multistate systems with multistate components, IEEE Trans. Comput. 52 (2003) 1608-1618.

[15] L. Xing, Y. Dai, A new decision-diagram-based method for efficient analysis on multistate systems, IEEE Trans. Dependable Secure Comput. 6 (2009) $161-174$.

[16] G. Levitin, L. Xing, S.V. Amari, Recursive algorithm for reliability evaluation of non-repairable phased mission systems with binary elements, IEEE Trans. Reliab. 61 (2012) 533-542.

[17] M. Alam, U.M. Al-Saggaf, Quantitative reliability evaluation of repairable phased-mission systems using Markov approach, IEEE Trans. Reliab. 35 (1986) 498-503.

[18] C. Wang, L. Xing, G. Levitin, Competing failure analysis in phased-mission systems with functional dependence in one of phases, Reliab. Eng. Syst. Saf. 108 (2012) 90-99.

[19] X. Wu, J. Hillston, Mission reliability of Semi-Markov systems under generalized operational time requirements, Reliab. Eng. Syst. Saf. 140 (2015) $122-129$.

[20] I. Mura, A. Bondavalli, Markov regenerative stochastic Petri nets to model and evaluate phased mission systems dependability, IEEE Trans. Commun. 50 (2001) 1337-1351.

[21] Y. Ou, J.B. Dugan, Modular solution of dynamic multi-phase systems, IEEE Trans. Reliab. 53 (2004) 499-508.

[22] W. Li, H. Pham, Reliability modeling of multi-state degraded systems with multi-competing failures and random shocks, IEEE Trans. Reliab. 54 (2005) 297-303.

[23] W. Peng, Y.-F. Li, Y.-J. Yang, J. Mi, H.-Z. Huang, Bayesian degradation analysis with inverse Gaussian process models under time-varying degradation rates, IEEE Trans. Reliab. 66 (2017) 84-96. 
[24] Y. Liu, H.-Z. Huang, Reliability assessment for fuzzy multi-state systems, Int. J. Syst. Sci. 41 (2010) 365-379.

[25] Y. Liu, H.-Z. Huang, Z. Wang, Y. Li, Y. Yang, A joint redundancy and imperfect maintenance strategy optimization for multi-state systems, IEEE Trans. Reliab. 62 (2013) 368-378.

[26] W. Peng, L. Shen, Y. Shen, Q. Sun, Reliability analysis of repairable systems with recurrent misuse-induced failures and normal-operation failures, Reliab. Eng. Syst. Saf. 171 (2018) 87-98.

[27] G. Levitin, The Universal Generating Function in Reliability Analysis and Optimization, Springer, London, 2005.

[28] Y. Liu, H.-Z. Huang, Optimal replacement policy for multi-state system under imperfect maintenance, IEEE Trans. Reliab. 59 (2010) 483-495.

[29] T. Jiang, Y. Liu, Parameter inference for non-repairable multi-state system reliability models by multi-level observation sequences, Reliab. Eng. Syst. Saf. 166 (2017) 3-15.

[30] W. Peng, N. Balakrishnan, H.-Z. Huang, Reliability modelling and assessment of a heterogeneously repaired system with partially relevant recurrence data, Appl. Math. Model. (2018).

[31] S.Y. Kim, J.-F. Castet, J.H. Saleh, Spacecraft electrical power subsystem: Failure behavior, reliability, and multi-state failure analyses, Reliab. Eng. Syst. Saf. 98 (2012) 55-65.

[32] J.-F. Castet, J.H. Saleh, Beyond reliability, multi-state failure analysis of satellite subsystems: a statistical approach, Reliab. Eng. Syst. Saf. 95 (2010) $311-322$.

[33] M. Carravetta, R. De Dominicis, M. D’Esposito, Semi-Markov processes in modelling and reliability: a new approach, Appl. Math. Model. 5 (1981) 269-274.

[34] S. Distefano, K.S. Trivedi, Non-Markovian state-space models in dependability evaluation, Qual. Reliab. Eng. Int. 29 (2013) $225-239$.

[35] W.Q. Meeker, L.A. Escobar, Statistical Methods For Reliability Data, John Wiley \& Sons, 2014.

[36] N.-C. Xiao, M.J. Zuo, W. Guo, Efficient reliability analysis based on adaptive sequential sampling design and cross-validation, Appl. Math. Model. 58 (2018) 404-420.

[37] E. Zio, The Monte Carlo Simulation Method For System Reliability and Risk Analysis, Springer, London, 2013.

[38] B. Keshtegar, O. Kisi, M5 model tree and Monte Carlo simulation for efficient structural reliability analysis, Appl. Math. Model. 48 (2017) 899-910. 